\title{
FIRST-ORDER SYSTEM LEAST SQUARES FOR SECOND-ORDER PARTIAL DIFFERENTIAL EQUATIONS: PART II*
}

\author{
ZHIQIANG $\mathrm{CAI}^{\dagger}$, THOMAS A. MANTEUFFEL ${ }^{\ddagger}$, AND STEPHEN F. MCCORMICK ${ }^{\S}$
}

\begin{abstract}
This paper develops a least-squares functional that arises from recasting general second-order uniformly elliptic partial differential equations in $n=2$ or 3 dimensions as a system of first-order equations. In part I [Z. Cai, R. D. Lazarov, T. Manteuffel, and S. McCormick, SIAM J. Numer. Anal., 31 (1994), pp. 1785-1799] a similar functional was developed and shown to be elliptic in the $H(\operatorname{div}) \times H^{1}$ norm and to yield optimal convergence for finite element subspaces of $H($ div $) \times H^{1}$. In this paper the functional is modified by adding a compatible constraint and imposing additional boundary conditions on the first-order system. The resulting functional is proved to be elliptic in the $\left(H^{1}\right)^{n+1}$ norm. This immediately implies optimal error estimates for finite element approximation by standard subspaces of $\left(H^{1}\right)^{n+1}$. Another direct consequence of this ellipticity is that multiplicative and additive multigrid algorithms applied to the resulting discrete functionals are optimally convergent. As an alternative to perturbation-based approaches, the least-squares approach developed here applies directly to convection-diffusion-reaction equations in a unified way and also admits a fast multigrid solver, historically a missing ingredient in least-squares methodology.
\end{abstract}

Key words. least-squares discretization, multigrid, second-order elliptic problems, iterative methods

AMS subject classifications. $65 \mathrm{~F} 10,65 \mathrm{~F} 30$

\section{PII. S0036142994266066}

1. Introduction. The object of study of this paper, and its earlier companion [11], is the solution of elliptic equations (including convection-diffusion and Helmholtz equations) by way of a least-squares formulation for an equivalent first-order system. Such formulations have been considered by several researchers over the last few decades (see the historical discussion in [11]), motivated in part by the possibility of a well-posed variational principle for a general class of problems. In [11] a similar functional was developed and shown to be elliptic in the $H($ div $) \times H^{1}$ norm and to yield optimal convergence for finite element subspaces of $H(\operatorname{div}) \times H^{1}$. In this paper the functional is modified by adding a compatible constraint and imposing additional boundary conditions on the first-order system. It is shown that the resulting functional is elliptic in the $\left(H^{1}\right)^{n+1}$ norm. Direct consequences of this result are optimal approximation error estimates for standard finite element subspaces of $\left(H^{1}\right)^{n+1}$ and optimal convergence of multiplicative and additive multigrid algorithms applied to the resulting discrete functionals. As an alternative to perturbation-based approaches (cf. $[1,3,9,10,25,34,35])$, the least-squares approach developed here applies directly to convection-diffusion-reaction equations in a unified way and also admits an efficient multilevel solver, historically a missing ingredient in least-squares methodology.

\footnotetext{
* Received by the editors April 5, 1994; accepted for publication (in revised form) May 3, 1995.

http://www.siam.org/journals/sinum/34-2/26606.html

${ }^{\dagger}$ Department of Mathematics, Purdue University, 1395 Mathematical Science Building, West Lafayette, IN 47907-1395 (zcai@math.purdue.edu). The work of this author was sponsored by the National Science Foundation grant DMS-9619792.

$\ddagger$ Program in Applied Mathematics, Campus Box 526, University of Colorado at Boulder, Boulder, CO 80309-0526 (tmanteuf@boulder.colorado.edu). The work of this author was sponsored by the National Science Foundation grant DMS-8704169 and Department of Energy grant DE-FG0393ER25217.

$\S$ Program in Applied Mathematics, Campus Box 526, University of Colorado at Boulder, Boulder, CO 80309-0526. The work of this author was sponsored by Air Force Office of Scientific Research grant AFOSR-86-0126, National Science Foundation grant DMS-8704169, and Department of Energy grant DE-FG03-93ER25165.
} 
The least-squares formulation considered in this paper differs from that of [11] (see also [31]) in that it incorporates a curl-free constraint and tangential boundary conditions on $\mathbf{u}$ (see section 2). Like the original in [11], our modified least-squares formulation avoids the so-called inf-sup condition of Ladyzhenskaya, Babǔska, and Brezzi (see [8]) and its attendant restrictions on the choice of finite element approximation subspaces. However, our modifications to the original form, while unnecessary for discretization accuracy (see [11]), greatly simplify the solution process. The unmodified form must be handled carefully because oscillatory divergence-free error components give relatively small residuals; such components must be specially treated in the multigrid relaxation process or eliminated in the discretization, for example. However, our incorporation of the curl-free constraint here exposes these components: in fact, all oscillatory error results in relatively large residuals. The modified functional is easily seen to be equivalent to a modified form of the $(H(\operatorname{div}) \cap H($ curl $)) \times H^{1}$ norm. This norm may be sufficient to yield optimal finite element convergence if care is taken in choosing the finite element spaces [33, 27]. In addition, optimal multigrid convergence can be guaranteed under certain additional constraints [27]. The theory involving this norm is still incomplete.

In this paper the modified $(H(\operatorname{div}) \cap H($ curl $)) \times H^{1}$ norm is shown to be equivalent to the $\left(H^{1}\right)^{n+1}$ norm under some additional regularity assumptions. Thus, the modified functional yields full $H^{1}$-norm equivalence for each of the system variables. This comes at some loss of generality of the results presented here in that our proof requires $H^{2}$ regularity of the original problem with the lower-order terms removed. This should be expected, however, since our aim here is to obtain $H^{1}$-norm discretization error bounds on the fluxes.

For problems with reduced regularity, alternate forms of the least-squares functional must be considered, with the specific choice of form dependent on the goal of computation: for $H$ (div)-type flux estimates, the original functional in [11] is appropriate; for weaker $L^{2}$-type flux estimates, for example when the right-hand side is in $H^{-1}$, an $H^{-1}$ or mesh-weighted norm approach can be used (cf. [2, 4]); and for stronger local $H^{1}$-type flux estimates, for example when the coefficients are discontinuous, an appropriate local $H^{1}$ approach can be used [27].

The basic idea of the approach here is simple: the original functional in [11], which fails to be fully $\left(H^{1}\right)^{n+1}$ elliptic because it incorporates only a divergence-type flux derivative, is augmented by an admissible curl-type term. It is therefore immediate that the new functional is equivalent to an $(H$ (div) $\cap H($ curl $)) \times H^{1}$-type norm. However, to show that such a norm is in fact an $\left(H^{1}\right)^{n+1}$ norm is another matter: for that (and only that!) we need $H^{2}$ regularity of the original scalar problem without the lower-order terms, and we need to carefully extend basic results of functional analysis to account for general diffusion tensors and boundary conditions. This is the principal theoretical contribution of this paper.

The idea of adding the curl constraint in developing a least-squares functional for Poisson's equation has been used by several researchers (see, e.g., [13, 16, 17, 23, 14, $15,24,30])$. For Poisson's equation, the key tool is the proof that $(H$ (div) $\cap H$ (curl)) is algebraically and topologically imbedded in $\left(H^{1}\right)^{n}$, which was developed by Girault and Raviart [20] for problems with strictly Dirichlet or Neumann boundary conditions. Here, we extend the result in [20] to the context of a general diffusion tensor and a variety of boundary conditions.

The fundamental goal of our least-squares work is to develop a functional that is fully $\left(H^{1}\right)^{n+1}$ elliptic whenever that is possible. One of the many benefits of such a 
formulation is that the discretization and solution processes can be designed for each variable individually, almost independently of the others. This ellipticity suggests that the system is essentially a set of uncoupled elliptic equations in each scalar variable. This is true to some qualitative degree, but this view should not be carried too far. For example, this equivalence implies immediately that an optimal multigrid solver can be designed simply as a diagonal preconditioner that uses multigrid individually on each variable. However, multigrid can generally do much better by applying it directly to the least-squares system: relaxation would be allowed to interact with all variables on all levels, not just on the finest as diagonal preconditioners would do.

Unfortunately, no theory seems to exist that would allow us to claim in any generality that such a fully integrated multigrid scheme is optimal. We are thus compelled to establish this claim theoretically, which we do in the last section by applying the theory developed in [5]. (The only nontrivial task here is the verification of their second smoothing assumption.)

The next section introduces notation, describes the variational approach, and establishes an ellipticity estimate in the necessary form. Approximation by the finite element method and its error analysis are developed in section 3.

2. First-order system least squares. Assume that $\Omega$ is a bounded, open, connected domain in $\Re^{n}(n=2$ or 3$)$ with Lipschitz boundary $\partial \Omega$. Consider the following second-order elliptic boundary value problem:

$$
\left\{\begin{aligned}
-\nabla \cdot(A \nabla p)+X p & =f, & & \text { in } \quad \Omega, \\
p & =0, & & \text { on } \quad \Gamma_{D}, \\
\mathbf{n} \cdot A \nabla p & =0, & & \text { on } \quad \Gamma_{N},
\end{aligned}\right.
$$

where the symbols $\nabla$. and $\nabla$ stand for the divergence and gradient operators, respectively, $A$ is an $n \times n$ symmetric matrix of functions in $L^{\infty}(\Omega), X$ is an at most first-order linear differential operator, $\Gamma_{D} \cup \Gamma_{N}=\Gamma$ is a partitioning of the boundary of $\Omega$, and $\mathbf{n}$ is the outward unit vector normal to the boundary. We assume that $A$ is uniformly symmetric positive definite and scaled appropriately: there exist positive constants

$$
0<\lambda \leq 1 \leq \Lambda
$$

such that

$$
\lambda \boldsymbol{\xi}^{T} \boldsymbol{\xi} \leq \boldsymbol{\xi}^{T} A \boldsymbol{\xi} \leq \Lambda \boldsymbol{\xi}^{T} \boldsymbol{\xi}
$$

for all $\boldsymbol{\xi} \in \Re^{n}$ and almost all $x \in \bar{\Omega}$.

Introducing the flux variable

$$
\mathbf{u}=A \nabla p,
$$

problem (2.1) may be rewritten as a first-order system of partial differential equations as follows:

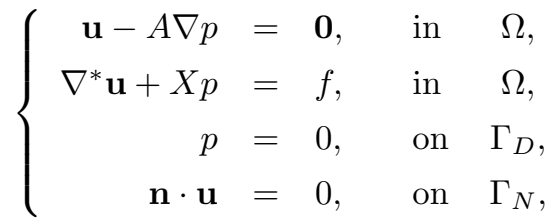


where $\nabla^{*}: H^{1}(\Omega)^{n} \rightarrow L^{2}(\Omega)$ is the formal adjoint of $\nabla: H^{1}(\Omega) \rightarrow L^{2}(\Omega)^{n}$; that is, $\nabla^{*} \equiv-\nabla \cdot$. Under appropriate assumptions on $\Gamma_{D}$ and $X$, the associated weak form of the system (2.1) is uniquely solvable in $H^{1}(\Omega)$ for any $f \in H^{-1}(\Omega)$ or uniquely solvable in $H^{1}(\Omega) / \Re$ (cf. [20]) if and only if $f$ satisfies the compatibility condition $\int_{\Omega} f=0$.

Let $\operatorname{curl} \equiv \nabla \times$ denote the curl operator. (Here and henceforth, we use notation for the case $n=3$ and consider the special case $n=2$ in the natural way by identifying $\Re^{2}$ with the $\left(x_{1}, x_{2}\right)$-plane in $\Re^{3}$. Thus, if $\mathbf{u}$ is two dimensional, then $\nabla \times \mathbf{u}=\mathbf{0}$ means $\partial_{1} u_{2}-\partial_{2} u_{1}=0$, where $u_{1}$ and $u_{2}$ are the components of $\mathbf{u}$. In section 2.1, we consider only the case $n=2$, so there we will interpret $\nabla \times \mathbf{u}$ to mean $\partial_{1} u_{2}-\partial_{2} u_{1}$.) Note that if $\mathbf{u}$ is sufficiently smooth, then the properly scaled solution $A^{-1} \mathbf{u}$ of (2.4) is curl free, i.e., $\nabla \times\left(A^{-1} \mathbf{u}\right)=\mathbf{0}$, and the homogeneous Dirichlet boundary condition on $\Gamma_{D}$ implies the tangential flux or no-slip condition

$$
\gamma_{\boldsymbol{\tau}}\left(A^{-1} \mathbf{u}\right)=\mathbf{0}, \quad \text { on } \Gamma_{D}
$$

where $\gamma_{\boldsymbol{\tau}} \mathbf{u} \equiv \mathbf{n} \times \mathbf{u}$. Here, $\boldsymbol{\tau}$ represents the unit vector tangent to the boundary $\Gamma$.

An equivalent extended system for (2.4) is

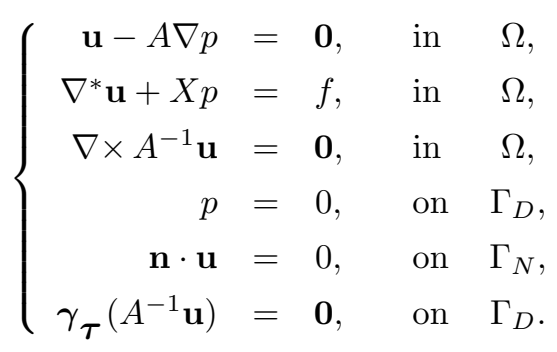

It is this system that we intend to solve by a least-squares Rayleigh-Ritz discretization and a fully variational multigrid solver.

First we establish notation. Let $\mathcal{D}(\Omega)$ be the linear space of infinitely differentiable functions with compact support on $\Omega$, and

$$
\mathcal{D}(\bar{\Omega})=\left\{\left.\varphi\right|_{\Omega}: \varphi \in \mathcal{D}(\mathcal{O}) \text { for some open subset } \Omega \subset \mathcal{O} \subset \Re^{n}\right\} .
$$

Let $(\cdot, \cdot)_{0, \Omega}$ denote the inner product on $L^{2}(\Omega)^{n},\|\cdot\|_{0, \Omega}$ denote its induced norm, and, for $m \geq 0, H^{m}(\Omega)^{n}$ denote the standard Sobolev space with norm $\|\cdot\|_{m, \Omega}$ and seminorms $|\cdot|_{i, \Omega}(0 \leq i \leq m)$. (We suppress the subscript $n$ because dependence of the vector norms on dimension will be clear by context.) Let $H^{s-\frac{1}{2}}(\Gamma)$ for $s=1$ or 2 denote the trace Sobolev space with norm

$$
\|r\|_{s-\frac{1}{2}, \Gamma}=\inf \left\{\|v\|_{s, \Omega}: v \in H^{s}(\Omega), \text { trace } v=r \text { on } \Gamma\right\}
$$

and $H^{-\frac{1}{2}}(\Gamma)$ the dual space for $H^{\frac{1}{2}}(\Gamma)$ with the obvious dual norm

$$
\left\|r^{*}\right\|_{-\frac{1}{2}, \Gamma}=\sup \left\{\frac{\left\langle r^{*}, r\right\rangle}{\|r\|_{1 / 2, \Gamma}}: 0 \neq r \in H^{\frac{1}{2}}(\Gamma)\right\}
$$

where $\langle\cdot, \cdot\rangle$ denotes the duality pairing between $H^{-\frac{1}{2}}(\Gamma)$ and $H^{\frac{1}{2}}(\Gamma)$. We use the following spaces to define a least-squares variational form for the extended system (2.5). Let

$$
H(\operatorname{div} ; \Omega)=\left\{\mathbf{v} \in L^{2}(\Omega)^{n}: \nabla^{*} \mathbf{v} \in L^{2}(\Omega)\right\}
$$




$$
H(\operatorname{curl} A ; \Omega)=\left\{\mathbf{v} \in L^{2}(\Omega)^{n}: \nabla \times\left(A^{-1} \mathbf{v}\right) \in L^{2}(\Omega)^{2 n-3}\right\},
$$

which are Hilbert spaces under the respective norms

$$
\begin{aligned}
\|\mathbf{v}\|_{H(\operatorname{div} ; \Omega)} & \equiv\left(\|\mathbf{v}\|_{0, \Omega}^{2}+\left\|\nabla^{*} \mathbf{v}\right\|_{0, \Omega}^{2}\right)^{\frac{1}{2}} \\
\|\mathbf{v}\|_{H(\operatorname{curl} A ; \Omega)} & \equiv\left(\|\mathbf{v}\|_{0, \Omega}^{2}+\left\|\nabla \times\left(A^{-1} \mathbf{v}\right)\right\|_{0, \Omega}^{2}\right)^{\frac{1}{2}} .
\end{aligned}
$$

When $A$ is the identity matrix in $(2.6)$, we use the simpler notation $H(\mathbf{c u r l} ; \Omega)$. Define the subspaces

$$
\begin{aligned}
\mathbf{W}_{0}(\operatorname{div} ; \Omega) & =\left\{\mathbf{v} \in H(\operatorname{div} ; \Omega): \mathbf{n} \cdot \mathbf{v}=\mathbf{0} \text { on } \Gamma_{N}\right\} \\
\mathbf{W}_{0}(\operatorname{curl} A ; \Omega) & =\left\{\mathbf{v} \in H(\operatorname{curl} A ; \Omega): \boldsymbol{\gamma}_{\boldsymbol{\tau}}\left(A^{-1} \mathbf{v}\right)=\mathbf{0} \text { on } \Gamma_{D}\right\},
\end{aligned}
$$

and

$$
\mathbf{W}=\mathbf{W}_{0}(\operatorname{div} ; \Omega) \cap \mathbf{W}_{0}(\operatorname{curl} A ; \Omega) .
$$

Finally, define the subspace

$$
V=\left\{q \in H^{1}(\Omega): q=0 \text { on } \Gamma_{D}\right\} .
$$

In [11] the following quadratic functional associated with system (2.4) was examined:

$$
G_{0}(\mathbf{v}, q ; f)=\|\mathbf{v}-A \nabla q\|_{0, \Omega}^{2}+\left\|\nabla^{*} \mathbf{v}+X q-f\right\|_{0, \Omega}^{2}
$$

for $(\mathbf{v}, q) \in \mathbf{W}_{0}(\operatorname{div} ; \Omega) \times V$. There it was shown that $G_{0}(\mathbf{v}, q ; 0)$ is equivalent to the $H(\operatorname{div} ; \Omega) \times H^{1}(\Omega)$ norm on $\mathbf{W}_{0}(\operatorname{div} ; \Omega) \times V$ under the following original assumption.

Assumption $A 0$. Either $\Gamma_{D} \neq \emptyset$ or an additional constraint is imposed on $V$, such as $\int_{\Omega} p d x=0$, so that a Poincaré-Friedrichs inequality holds: there exists a constant $d>0$ depending only on the domain $\Omega$ and the uniform bounds on $A$ (see (2.3)) such that

$$
\|p\|_{0, \Omega}^{2} \leq d\left\|A^{\frac{1}{2}} \nabla p\right\|_{0, \Omega}^{2}
$$

for $p \in V$.

If $\Gamma_{D} \neq \emptyset$ or $X p \neq 0$ for $p \equiv$ constant, we assume that for any $f \in H^{-1}(\Omega)$ the associated weak form of $(2.1)$ is invertible in $H^{1}(\Omega)$. If $\Gamma_{D}=\emptyset$ and $X p=0$ for $p \equiv$ constant, we assume that the associated weak form of (2.1) is invertible in $H^{1}(\Omega) / \Re$ for every $f \in H^{-1}(\Omega)$ such that $\int_{\Omega} f=0$. In either case, we assume

$$
\|X p\|_{0, \Omega} \leq \eta\left\|A^{\frac{1}{2}} \nabla p\right\|_{0, \Omega}
$$

for some $\eta>0$ and every $p \in V$ for which $A \nabla p \in \mathbf{W}_{0}($ div $; \Omega)$.

The modified quadratic functional we study here is given by

$$
G(\mathbf{v}, q ; f)=\|\mathbf{v}-A \nabla q\|_{0, \Omega}^{2}+\left\|\nabla^{*} \mathbf{v}+X q-f\right\|_{0, \Omega}^{2}+\left\|\nabla \times\left(A^{-1} \mathbf{v}\right)\right\|_{0, \Omega}^{2}
$$

for $(\mathbf{v}, q) \in \mathbf{W} \times V$. Then the least-squares problem for (2.5) is to minimize this quadratic functional over $\mathbf{W} \times V$ : find $(\mathbf{u}, p) \in \mathbf{W} \times V$ such that

$$
G(\mathbf{u}, p ; f)=\inf _{(\mathbf{v}, q) \in \mathbf{W} \times V} G(\mathbf{v}, q ; f) .
$$


It is easy to see that the variational form for (2.13) is to find $(\mathbf{u}, p) \in \mathbf{W} \times V$ such that

$$
\mathcal{F}(\mathbf{u}, p ; \mathbf{v}, q)=f(\mathbf{v}, q) \quad \forall(\mathbf{v}, q) \in \mathbf{W} \times V,
$$

where the bilinear form $\mathcal{F}(\cdot ; \cdot):(\mathbf{W} \times V)^{2} \longrightarrow \Re$ is defined by

$$
\begin{aligned}
\mathcal{F}(\mathbf{u}, p ; \mathbf{v}, q) & =(\mathbf{u}-A \nabla p, \mathbf{v}-A \nabla q)_{0, \Omega}+\left(\nabla^{*} \mathbf{u}+X p, \nabla^{*} \mathbf{v}+X q\right)_{0, \Omega} \\
& +\left(\nabla \times\left(A^{-1} \mathbf{u}\right), \nabla \times\left(A^{-1} \mathbf{v}\right)\right)_{0, \Omega}
\end{aligned}
$$

and the linear functional $f(\cdot, \cdot): \mathbf{W} \times V \longrightarrow \Re$ is defined by

$$
f(\mathbf{v}, q)=\left(f, \nabla^{*} \mathbf{v}+X q\right)_{0, \Omega} .
$$

The first theorem establishes ellipticity and continuity of the bilinear form (2.15) with respect to the $(H(\operatorname{div} ; \Omega) \cap H(\operatorname{curl} A ; \Omega)) \times H^{1}(\Omega)$ norm under only Assumption $A 0$.

THEOREM 2.1. Assume A0. Then there exist positive constants $\alpha_{0}$ and $\alpha_{1}$ such that

$$
\alpha_{0}\left(\|\mathbf{v}\|_{0, \Omega}^{2}+\left\|\nabla^{*} \mathbf{v}\right\|_{0, \Omega}^{2}+\left\|\nabla \times\left(A^{-1} \mathbf{v}\right)\right\|_{0, \Omega}^{2}+\|q\|_{1, \Omega}^{2}\right) \leq \mathcal{F}(\mathbf{v}, q ; \mathbf{v}, q)
$$

for any $(\mathbf{v}, q) \in \mathbf{W} \times V$ and

$$
\begin{aligned}
\mathcal{F}(\mathbf{u}, p ; \mathbf{v}, q) \leq \alpha_{1}\left(\|\mathbf{u}\|_{0, \Omega}^{2}+\left\|\nabla^{*} \mathbf{u}\right\|_{0, \Omega}^{2}+\left\|\nabla \times\left(A^{-1} \mathbf{u}\right)\right\|_{0, \Omega}^{2}+\|p\|_{1, \Omega}^{2}\right)^{\frac{1}{2}} \\
\cdot\left(\|\mathbf{v}\|_{0, \Omega}^{2}+\left\|\nabla^{*} \mathbf{v}\right\|_{0, \Omega}^{2}+\left\|\nabla \times\left(A^{-1} \mathbf{v}\right)\right\|_{0, \Omega}^{2}+\|q\|_{1, \Omega}^{2}\right)^{\frac{1}{2}}
\end{aligned}
$$

for any $(\mathbf{u}, p),(\mathbf{v}, q) \in \mathbf{W} \times V$.

Proof. This result follows from Theorem 3.1 in [11], where it was shown that the functional

$$
G_{0}(\mathbf{v}, q ; f)=\|\mathbf{v}-A \nabla q\|_{0, \Omega}^{2}+\left\|\nabla^{*} \mathbf{v}+X q-f\right\|_{0, \Omega}^{2}
$$

and associated bilinear form

$$
\mathcal{F}_{0}(\mathbf{u}, p ; \mathbf{v}, q)=(\mathbf{u}-A \nabla p, \mathbf{v}-A \nabla q)_{0, \Omega}+\left(\nabla^{*} \mathbf{u}+X p, \nabla^{*} \mathbf{v}+X q\right)_{0, \Omega}
$$

satisfy the bounds (2.17) and (2.18) with the terms involving $\nabla \times$ removed, for all $(\mathbf{u}, p),(\mathbf{v}, q) \in \mathbf{W}_{0}(\operatorname{div} ; \Omega) \times V$. Since $\mathbf{W} \subset \mathbf{W}_{0}(\operatorname{div} ; \Omega),(2.17)$ and (2.18) hold for $\mathcal{F}$ restricted to $\mathbf{W} \times V$.

The purpose of the remainder of this section is to show that, under some additional hypotheses on $A$ and $\Omega$, the functional (2.12) with $f=0$ is equivalent to the $H^{1}(\Omega)^{n+1}$ norm on $\mathbf{W} \times V$. This result requires a generalization of results in [20], which assumed that $A=I, X=0$, and either $\Gamma=\Gamma_{D}$ or $\Gamma=\Gamma_{N}$.

In our proof we make use of a decomposition of $\mathbf{W}$ into certain gradient and curl components. We do this by showing that these components satisfy certain boundary value problems, which we ensure are $H^{2}(\Omega)$ regular by making the following additional assumptions.

Assumption A1. The domain $\Omega$ is bounded, open, and connected in $\Re^{n}(n=2$ or 3) with boundary $\Gamma$, which consists of a finite number of disjoint, simple, closed 


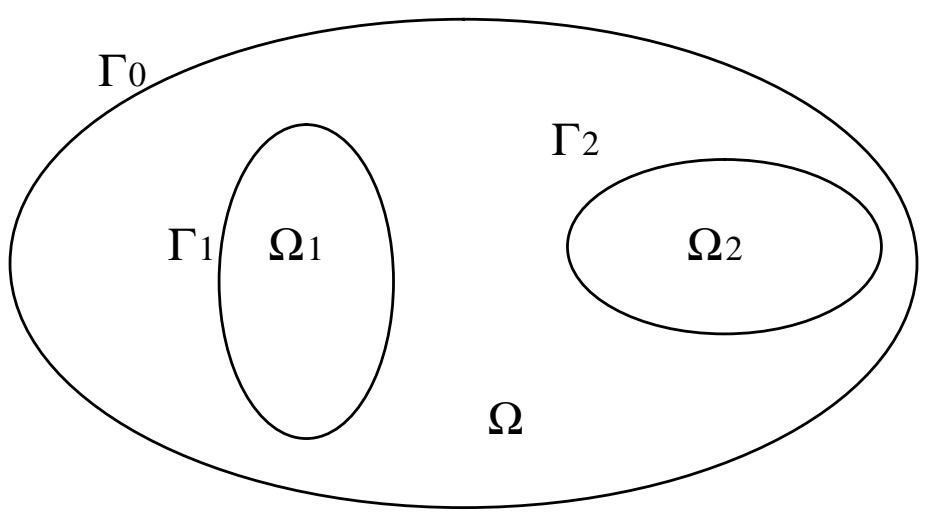

FIG. 1.

curves (surfaces) $\Gamma_{i}, i=0, \ldots, L ; \Gamma_{0}$ is the outer boundary and $\Gamma_{i}, i=1, \ldots, L$, are $C^{1,1}$ boundaries of a finite number of disjoint holes in $\Omega$ (see Fig. 1). Let $\Omega_{i}$ be the interior of $\Gamma_{i}, i=1, \ldots, L$. For $n=2, \Gamma_{0}$ is piecewise $C^{1,1}$ with no reentrant corners, while for $n=3, \Gamma_{0}$ is $C^{1,1}$ or a convex polyhedron.

Assumption A2. The boundary is divided into Dirichlet and Neumann parts: $\Gamma=$ $\Gamma_{D} \cup \Gamma_{N}$ such that $\Gamma_{i} \subseteq \Gamma_{D}$ for $i \in D$ and $\Gamma_{i} \subseteq \Gamma_{N}$ for $i \in N$ with $D \cup N=\{1, \ldots, L\}$. For $n=2, \Gamma_{0}$ is divided into a finite number of connected pieces: $\Gamma_{0}=\cup_{i=1, \ldots, M} \Gamma_{0, i}$ such that $\Gamma_{0, i} \subseteq \Gamma_{D}$ for $i \in D_{0}$ and $\Gamma_{0, i} \subseteq \Gamma_{N}$ for $i \in N_{0}$; since $\Gamma_{0}$ is a simple closed curve, $M$ is even; let $D_{0}$ be the odd indices and $N_{0}$ be the even indices. For $n=3$, either $\Gamma_{0} \subseteq \Gamma_{D}$ or $\Gamma_{0} \subseteq \Gamma_{N}$.

Assumption A3. The matrix $A$ is $C^{1,1}$. If $n=2$ and $\mathbf{x} \in \Gamma_{0}$ is a point that separates $\Gamma_{D}$ and $\Gamma_{N}$, then $\mathbf{x}$ must be a corner of $\Gamma_{0}$ and $\mathbf{n}_{-}^{T} A \mathbf{n}_{+} \leq 0$, where $\mathbf{n}_{-}$and $\mathbf{n}_{+}$are the outward unit normal vectors on the adjacent edges at $\underline{\mathbf{x}}$.

In what follows, we will appeal often to a boundary value problem of the form

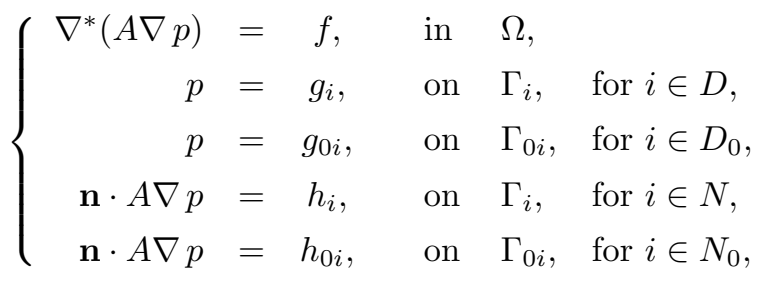

where $g_{i}, g_{0 j} \in H^{\frac{3}{2}}\left(\Gamma_{i}\right)$ for $i \in D, j \in D_{0}$, and $h_{i}, h_{0 j} \in H^{\frac{1}{2}}\left(\Gamma_{i}\right)$ for $i \in N, j \in N_{0}$. For our needs, we assume that $g_{i}$ is nonzero only when $\Gamma_{i}$ is $C^{1,1}$. Now our additional assumptions, together with the original ones, are sufficient to guarantee that (2.19) is $H^{2}(\Omega)$ regular: there exists a constant $C$ depending only on $A, X$, and $\Omega$ such that

$$
\begin{aligned}
\|p\|_{2, \Omega} \leq C\left(\|f\|_{0, \Omega}\right. & +\sum_{i \in D}\left\|g_{i}\right\|_{3 / 2, \Gamma_{i}}+\sum_{i \in D_{0}}\left\|g_{0 i}\right\|_{3 / 2, \Gamma_{i}}+\sum_{i \in N}\left\|h_{i}\right\|_{1 / 2, \Gamma_{i}} \\
& \left.+\sum_{i \in N_{0}}\left\|h_{0 i}\right\|_{1 / 2, \Gamma_{i}}\right) .
\end{aligned}
$$

This result follows from standard partition of unity arguments (cf. [19]). For both $n=2$ and $n=3$, the solution is clearly in $H^{2}$ in the interior and along smooth 
portions of the boundary. For $n=2$ the arguments in Chapter 4 of Grisvard [21] can be used near the corners. There, polygonal domains are studied with $A=I$ and $H^{2}$ regularity results if there are no reentrant corners and each corner that separates $\Gamma_{D}$ from $\Gamma_{N}$ has an angle less than $\pi / 2$. In our context, we consider $A \in C^{1,1}$. Since $A$ is smooth, there exists a smooth transformation to a problem in a new coordinate system with $A$ replaced by $I$. The results of Grisvard can be applied in this frame and the inverse transformation yields the criterion in Assumption $A 3$. For $n=3$, $\Gamma_{0}$ is either $C^{1,1}$, which poses no problem or $\Gamma_{0}$ is a convex polyhedron. Since by assumption $\Gamma_{0} \subseteq \Gamma_{D}$ or $\Gamma_{0} \subseteq \Gamma_{N}$ the results in Chapter 8 of [21] imply that a convex polyhedron is sufficient.

We remark that the results in this paper are applicable to any domains for which problems of the type $(2.19)$ are $H^{2}(\Omega)$ regular. Our assumptions reflect the limit of current knowledge in this respect.

Our main theorem establishes equivalence of the bilinear form (2.15) and the $H^{1}(\Omega)^{n+1}$ norm under the additional assumptions $A 1-A 3$.

THEOREM 2.2. Assume A0-A3. Then there exist positive constants $\alpha_{2}$ and $\alpha_{3}$ such that

$$
\alpha_{2}\left(\|\mathbf{v}\|_{1, \Omega}^{2}+\|q\|_{1, \Omega}^{2}\right) \leq \mathcal{F}(\mathbf{v}, q ; \mathbf{v}, q)
$$

for any $(\mathbf{v}, q) \in \mathbf{W} \times V$ and

$$
\mathcal{F}(\mathbf{u}, p ; \mathbf{v}, q) \leq \alpha_{3}\left(\|\mathbf{u}\|_{1, \Omega}^{2}+\|p\|_{1, \Omega}^{2}\right)^{\frac{1}{2}}\left(\|\mathbf{v}\|_{1, \Omega}^{2}+\|q\|_{1, \Omega}^{2}\right)^{\frac{1}{2}}
$$

for any $(\mathbf{u}, p),(\mathbf{v}, q) \in \mathbf{W} \times V$.

Proof. In light of Theorem 2.1, to prove Theorem 2.2 we need only show that $\mathbf{W}$ is algebraically and topologically included in $H^{1}(\Omega)^{n}$; that is, for any $\mathbf{v} \in \mathbf{W}$ there exist constants $\hat{\alpha}_{2}$ and $\hat{\alpha}_{3}$ such that

$$
\hat{\alpha}_{2}\|\mathbf{v}\|_{1, \Omega}^{2} \leq\|\mathbf{v}\|_{0, \Omega}^{2}+\left\|\nabla^{*} \mathbf{v}\right\|_{0, \Omega}^{2}+\left\|\nabla \times A^{-1} \mathbf{v}\right\|_{0, \Omega}^{2} \leq \hat{\alpha}_{3}\|\mathbf{v}\|_{1, \Omega}^{2} .
$$

The upper bound in (2.23) follows from the triangle inequality. We prove the lower bound for $n=2$ and 3 separately. The two-dimensional result could have been deduced as a special case of the three-dimensional result, but it would then have inherited the more restrictive assumptions.

2.1. Two dimensions. In this section, we interpret the curl of a vector function $\mathbf{u}$ to mean the scalar function $\nabla \times \mathbf{u}=\partial_{1} u_{2}-\partial_{2} u_{1}$. Note that, for $n=2$, the operator $\nabla^{\perp}$ defined by

$$
\nabla^{\perp} q \equiv\left(\begin{array}{cc}
0 & 1 \\
-1 & 0
\end{array}\right) \nabla q=\left(\begin{array}{c}
\partial_{2} q \\
-\partial_{1} q
\end{array}\right)
$$

is the formal adjoint of $\nabla \times$ :

$$
\nabla \times \mathbf{v}=\nabla^{*}\left(\begin{array}{rr}
0 & -1 \\
1 & 0
\end{array}\right) \mathbf{v} .
$$

Let

$$
P=\left(\begin{array}{cc}
0 & 1 \\
-1 & 0
\end{array}\right)
$$


then

$$
\begin{aligned}
& P \nabla=\nabla^{\perp}, \quad P^{*} \nabla^{\perp}=\nabla, \\
& \nabla^{*} P^{*}=\nabla \times, \quad \nabla \times P=\nabla^{*} .
\end{aligned}
$$

Let $\mathbf{n}=\left(n_{1}, n_{2}\right)^{t}$ be the outward unit normal and let $\boldsymbol{\tau}=\left(\tau_{1}, \tau_{2}\right)^{t}$ be the unit tangent oriented clockwise on $\Gamma_{0}$. Then $\boldsymbol{\tau}=P \mathbf{n}$. Many general results involving $\nabla^{*}$ and $\nabla$ can be restated for $\nabla \times$ and $\nabla^{\perp}$ by using $P$. In [20] we find the following result.

LEMMA 2.1. Let $\Omega \subset \Re^{2}$; then $\mathbf{w} \in H(\operatorname{div} ; \Omega)$ such that $\nabla^{*} \mathbf{w}=0$ and $\int_{\Gamma_{i}} \mathbf{n} \cdot \mathbf{w}=$ 0 for $i=1, \ldots, L$ if and only if $\mathbf{w}=\nabla^{\perp} q$ with $q \in H^{1}(\Omega)$.

Proof. See Theorem 3.1 in Chapter I of [20].

This becomes the following.

LEMMA 2.2. Let $\Omega \subset \Re^{2}$; then $\mathbf{w} \in H(\operatorname{curl} ; \Omega)$ such that $\nabla \times \mathbf{w}=0$ and $\int_{\Gamma_{i}} \boldsymbol{\tau} \cdot \mathbf{w}=0$ for $i=1, \ldots, L$ if and only if $\mathbf{w}=\nabla q$ with $q \in H^{1}(\Omega)$.

Proof. The proof follows from Lemma 2.1 and (2.24).

A result analogous to Green's formula also follows:

$$
(\nabla \times \mathbf{z}, \phi)=\left(\mathbf{z}, \nabla^{\perp} \phi\right)-\int_{\Gamma}(\boldsymbol{\tau} \cdot \mathbf{z}) \phi
$$

for $\mathbf{z} \in H(\operatorname{curl} ; \Omega)$ and $\phi \in H^{1}(\Omega)$.

The next lemma obtains sufficient conditions for a vector function in $\mathbf{W}$ to be zero.

LEMMA 2.3. Let $A$ be uniformly symmetric positive definite on $\Omega$, which satisfies Assumptions $A 1$ and $A 2$. Let $\mathbf{z} \in \mathbf{W}$ satisfy and either

or

vi)

$$
\begin{aligned}
\nabla^{*} \mathbf{z} & =0, \quad \text { in } \Omega, \\
\nabla \times A^{-1} \mathbf{z} & =0, \quad \text { in } \Omega, \\
\int_{\Gamma_{i}} \mathbf{n} \cdot \mathbf{z} & =0, \quad \text { for } i \in D, \\
\int_{\Gamma_{i}} \boldsymbol{\tau} \cdot A^{-1} \mathbf{z} & =0, \quad \text { for } i \in N,
\end{aligned}
$$

)

$$
\int_{\Gamma_{0 j}} \mathbf{n} \cdot \mathbf{z}=0, \quad \text { for } j \in D_{0},
$$

$$
\int_{\Gamma_{0 j}} \boldsymbol{\tau} \cdot A^{-1} \mathbf{z}=0, \text { for } j \in N_{0}
$$

Then $\mathbf{z}=\mathbf{0}$.

Proof. Assumptions (2.26) i), ii), iii), and iv) together with Lemmas 2.1 and 2.2 yield

$$
\mathbf{z}=A \nabla p, \quad \mathbf{z}=\nabla^{\perp} \phi
$$

with $p, \phi \in H^{1}(\Omega)$. Using Green's formula and assumption i), we have

$$
\begin{aligned}
\left(\nabla^{*} \mathbf{z}, p\right) & =(\mathbf{z}, \nabla p)-\int_{\Gamma}(\mathbf{n} \cdot \mathbf{z}) p \\
& =\left(A^{-1} \mathbf{z}, \mathbf{z}\right)-\int_{\Gamma}(\mathbf{n} \cdot \mathbf{z}) p=0 .
\end{aligned}
$$

Thus,

$$
\left(A^{-1} \mathbf{z}, \mathbf{z}\right)=\int_{\Gamma_{0}}(\mathbf{n} \cdot \mathbf{z}) p+\sum_{i \in D} \int_{\Gamma_{i}}(\mathbf{n} \cdot \mathbf{z}) p+\sum_{i \in N} \int_{\Gamma_{i}}(\mathbf{n} \cdot \mathbf{z}) p .
$$


The last sum is zero because $\mathbf{n} \cdot \mathbf{z}=0$ on $\Gamma_{N}$. The second sum is also zero because integration by parts on each of its terms yields

$$
\int_{\Gamma_{i}}(\mathbf{n} \cdot \mathbf{z}) p=-\int_{\Gamma_{i}}(\boldsymbol{\tau} \cdot \nabla \phi) p=\int_{\Gamma_{i}}(\boldsymbol{\tau} \cdot \nabla p) \phi=\int_{\Gamma_{i}}\left(\boldsymbol{\tau} \cdot A^{-1} \mathbf{z}\right) \phi=0,
$$

since the integration is around a closed path and $\boldsymbol{\tau} \cdot A^{-1} \mathbf{z}=0$ on $\Gamma_{D}$.

To prove that the first term on the right-hand side of (2.27) is also zero, assume first that (2.26) v) holds. Since $\mathbf{z} \in \mathbf{W}$, then $\mathbf{n} \cdot \mathbf{z}=\mathbf{0}$ on $\Gamma_{N}$ and we have

$$
\begin{aligned}
\int_{\Gamma_{0}}(\mathbf{n} \cdot \mathbf{z}) p & =\sum_{j \in D_{0}} \int_{\Gamma_{0 j}}(\mathbf{n} \cdot \mathbf{z}) p+\sum_{j \in N_{0}} \int_{\Gamma_{0 j}}(\mathbf{n} \cdot \mathbf{z}) p \\
& =\sum_{j \in D_{0}} \int_{\Gamma_{0 j}}(\mathbf{n} \cdot \mathbf{z}) p .
\end{aligned}
$$

Using this relation and noting that $\boldsymbol{\tau} \cdot A^{-1} \mathbf{z}=\boldsymbol{\tau} \cdot \nabla p=0$ on $\Gamma_{D}$, which implies that $p=\alpha_{j}$ on $\Gamma_{0 j}$ for $j \in D_{0}$ and some constant $\alpha_{j}$, we have

$$
\int_{\Gamma_{0}}(\mathbf{n} \cdot \mathbf{z}) p=\sum_{j \in D_{0}} \alpha_{j} \int_{\Gamma_{0 j}} \mathbf{n} \cdot \mathbf{z}=0
$$

by assumption v).

Next, assume that case (2.26) vi) holds. Since the integration is over a closed path, and $\boldsymbol{\tau} \cdot \nabla p=\boldsymbol{\tau} \cdot A^{-1} \mathbf{z}=0$ on $\Gamma_{D}$, we have

$$
\begin{aligned}
\int_{\Gamma_{0}}(\mathbf{n} \cdot \mathbf{z}) p & =-\int_{\Gamma_{0}}(\boldsymbol{\tau} \cdot \nabla \phi) p \\
& =\int_{\Gamma_{0}}(\boldsymbol{\tau} \cdot \nabla p) \phi \\
& =\sum_{j \in D_{0}} \int_{\Gamma_{0 j}}(\boldsymbol{\tau} \cdot \nabla p) \phi+\sum_{j \in N_{0}} \int_{\Gamma_{0 j}}(\boldsymbol{\tau} \cdot \nabla p) \phi \\
& =\sum_{j \in N_{0}} \int_{\Gamma_{0 j}}(\boldsymbol{\tau} \cdot \nabla p) \phi .
\end{aligned}
$$

Similar to the above, using this relation and noting that $\mathbf{n} \cdot \mathbf{z}=-\boldsymbol{\tau} \cdot \nabla \phi=0$ on $\Gamma_{N}$, which implies that $\phi=\beta_{j}$ on $\Gamma_{0 j}$ for $j \in N_{0}$ and some constant $\beta_{j}$, we have

$$
\int_{\Gamma_{0}}(\mathbf{n} \cdot \mathbf{z}) p=\sum_{j \in N_{0}} \beta_{j} \int_{\Gamma_{0 j}} \boldsymbol{\tau} \cdot A^{-1} \mathbf{z}=0
$$

by assumption vi).

In either case, we thus have

$$
\left(A^{-1} \mathbf{z}, \mathbf{z}\right)=0 .
$$

Since $A$ is uniformly symmetric positive definite, it follows that $\mathbf{z}=\mathbf{0}$.

We now construct a basis for the functions in $\mathbf{W}$ that satisfy (2.26) i) and ii). Consider the functions $p_{i}$ for $i \in D$ that satisfy

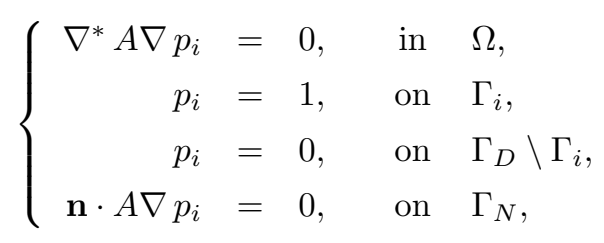


and the functions $p_{0 j}$ for $j \in D_{0}$ that satisfy

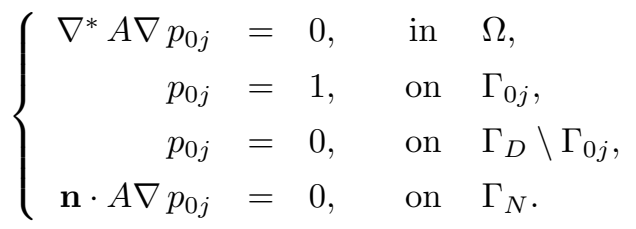

Clearly, $A \nabla p_{i}, A \nabla p_{0 j} \in \mathbf{W}$, and they satisfy (2.26) i) and ii). Suppose that Assumptions $A 1-A 3$ hold. Then we can recast (2.29) so that it has homogeneous boundary data but a nonzero source term bounded in $L^{2}(\Omega)$ by a constant depending only on $\Omega$. (This is easily done by extending the special Dirichlet data of (2.29) smoothly into $\Omega$ while satisfying the homogeneous Neumann boundary conditions, then restating (2.29) as an equation for the difference.) Thus, (2.20) applies so that both (2.28) and $(2.29)$ are $H^{2}(\Omega)$ regular and

$$
\left\|p_{i}\right\|_{2, \Omega} \leq C_{i}, \quad\left\|p_{0 j}\right\|_{2, \Omega} \leq C_{0 j},
$$

for $i \in D$ and $j \in D_{0}$, where $C_{i}$ and $C_{0 j}$ depend only on $\Omega$ and $A$.

Next, note that

$$
\nabla \times A^{-1} \nabla^{\perp}=\nabla^{*} P^{*} A^{-1} P \nabla=\nabla^{*} B \nabla,
$$

where

$$
B \equiv P^{*} A^{-1} P=\frac{1}{\operatorname{det}(A)} A .
$$

This relation is easily verified algebraically for any $2 \times 2$ symmetric matrix $A$. Now $B$ is uniformly symmetric positive definite:

$$
\frac{1}{\Lambda} \boldsymbol{\xi}^{T} \boldsymbol{\xi} \leq \boldsymbol{\xi}^{T} B \boldsymbol{\xi} \leq \frac{1}{\lambda} \boldsymbol{\xi}^{T} \boldsymbol{\xi}
$$

for all $\boldsymbol{\xi} \in R^{n}$ and $\mathbf{x} \in \bar{\Omega}$. Consider the functions $\phi_{i}$ for $i \in N$ that satisfy

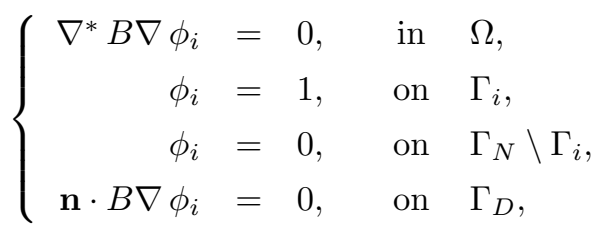

and the functions $\phi_{0 j}$ for $j \in N_{0}$ that satisfy

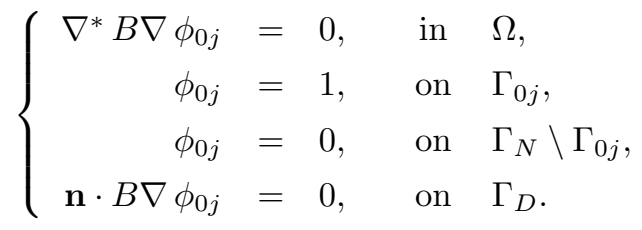

Since

$$
\begin{aligned}
\boldsymbol{\tau} \cdot A^{-1} \nabla^{\perp} \phi_{i} & =\mathbf{n} \cdot B \nabla \phi_{i}=0, & & \text { on } \Gamma_{D}, \\
\mathbf{n} \cdot \nabla^{\perp} \phi_{i} & =\boldsymbol{\tau} \cdot \nabla \phi_{i}=0, & & \text { on } \Gamma_{N}, \\
\boldsymbol{\tau} \cdot A^{-1} \nabla^{\perp} \phi_{0 j} & =\mathbf{n} \cdot B \nabla \phi_{0 j}=0, & & \text { on } \Gamma_{D}, \\
\mathbf{n} \cdot \nabla^{\perp} \phi_{0 j} & =\boldsymbol{\tau} \cdot \nabla \phi_{0 j}=0, & & \text { on } \Gamma_{N},
\end{aligned}
$$


it follows from (2.31), (2.33), and (2.34) that $\nabla^{\perp} \phi_{i}, \nabla^{\perp} \phi_{0 j} \in \mathbf{W}$, and that they satisfy (2.26) i) and ii). We note that if Assumptions $A 1-A 3$ hold, then problems (2.33) and (2.34) satisfy our additional assumptions: the uniformly symmetric positive definite matrix $B$ is $C^{1,1}$ and, if $\underline{x} \in \Gamma_{0}$ separates $\Gamma_{N}$ and $\Gamma_{D}$, then

$$
\mathbf{n}_{-}^{T} B \mathbf{n}_{+}=\frac{1}{\operatorname{det}(A)} \mathbf{n}_{-}^{T} A \mathbf{n}_{+} \leq 0 .
$$

Thus, since (2.20) applies as before, then both (2.33) and (2.34) are $H^{2}(\Omega)$ regular and there exist constants $C_{i}^{\prime}$ and $C_{0 j}^{\prime}$ such that

$$
\left\|\phi_{i}\right\|_{2, \Omega} \leq C_{i}^{\prime}, \quad\left\|\phi_{0 j}^{\prime}\right\|_{2, \Omega} \leq C_{0 j}^{\prime}
$$

for $i \in N$ and $j \in N_{0}$, where $C_{i}^{\prime}$ and $C_{0 j}^{\prime}$ depend only on $\Omega$ and $A$.

LEMMA 2.4. Let $A$ be $C^{1,1}$ and uniformly symmetric positive definite on $\Omega$, which satisfies Assumptions $A 1$ and $A 2$. Let $\mathbf{z} \in \mathbf{W}$ satisfy

$$
\text { i) } \begin{aligned}
\nabla^{*} \mathbf{z} & =0, \quad \text { in } \Omega, \\
\text { ii) } \nabla \times A^{-1} \mathbf{z} & =0, \quad \text { in } \Omega .
\end{aligned}
$$

Then

$$
\mathbf{z}=\sum_{j \in D_{0}} \alpha_{0 j} A \nabla p_{0 j}+\sum_{i \in D} \alpha_{i} A \nabla p_{i}+\sum_{j \in N_{0}} \beta_{0 j} \nabla^{\perp} \phi_{0 j}+\sum_{i \in N} \beta_{i} \nabla^{\perp} \phi_{i}
$$

Moreover, if Assumption A3 holds, then $\mathbf{z} \in H^{1}(\Omega)^{2}$ and

$$
\|\mathbf{z}\|_{1, \Omega} \leq C\|\mathbf{z}\|_{0, \Omega}
$$

where $C$ depends only on $\Omega$ and $A$.

Proof. We begin by constructing a function $\mathbf{w}$ in one of two ways:

$$
\mathbf{w}=\mathbf{z}-\left(\sum_{j \in D_{0}} \alpha_{0 j} A \nabla p_{0 j}+\sum_{i \in D} \alpha_{i} A \nabla p_{i}+\sum_{i \in N} \beta_{i} \nabla^{\perp} \phi_{i}\right)
$$

or

$$
\mathbf{w}=\mathbf{z}-\left(\sum_{i \in D} \alpha_{i} A \nabla p_{i}+\sum_{j \in N_{0}} \beta_{0 j} \nabla^{\perp} \phi_{0 j}+\sum_{i \in N} \beta_{i} \nabla^{\perp} \phi_{i}\right) .
$$

We will then show that proper choices of coefficients lead to the conclusion that $\mathbf{w}=\mathbf{0}$. To this end, choose $\alpha_{0 j}, \alpha_{i}, \beta_{0 j}$, and $\beta_{i}$ so that

$$
\int_{\Gamma_{i}} \mathbf{n} \cdot \mathbf{w}=0, \quad i \in D, \quad \int_{\Gamma_{i}} \boldsymbol{\tau} \cdot A^{-1} \mathbf{w}=0, \quad i \in N
$$

and

$$
\text { either } \int_{\Gamma_{0 j}} \mathbf{n} \cdot \mathbf{w}=0, \quad j \in D_{0}, \quad \text { or } \quad \int_{\Gamma_{0 j}} \boldsymbol{\tau} \cdot A^{-1} \mathbf{w}=0, \quad j \in N_{0}
$$


A decomposition of the form (2.39) is accomplished by solving the linear system

$$
\begin{aligned}
& \sum_{j \in D_{0}}\left(\int_{\Gamma_{0 i}} \mathbf{n} \cdot A \nabla p_{0 j}\right) \alpha_{0 j} \quad+\sum_{j \in D}\left(\int_{\Gamma_{0 i}} \mathbf{n} \cdot A \nabla p_{j}\right) \alpha_{j} \quad+\sum_{j \in N}\left(\int_{\Gamma_{0 i}} \mathbf{n} \cdot \nabla^{\perp} \phi_{j}\right) \beta_{j} \quad=\int_{\Gamma_{0 i}} \mathbf{n} \cdot \mathbf{z} \quad \text { for } i \in D_{0}, \\
& \sum_{j \in D_{0}}\left(\int_{\Gamma_{i}} \mathbf{n} \cdot A \nabla p_{0 j}\right) \alpha_{0 j} \quad+\sum_{j \in D}\left(\int_{\Gamma_{i}} \mathbf{n} \cdot A \nabla p_{j}\right) \alpha_{j} \quad=\int_{\Gamma_{i}} \mathbf{n} \cdot \mathbf{z} \quad \text { for } i \in D, \\
& \sum_{j \in D_{0}}\left(\int_{\Gamma_{i}} \tau \cdot \nabla p_{0 j}\right) \alpha_{0 j} \quad+\sum_{j \in N}\left(\int_{\Gamma_{i}} \mathbf{n} \cdot B \nabla \phi_{j}\right) \beta_{j} \quad=\int_{\Gamma_{i}} \tau \cdot A^{-1} \mathbf{z} \quad \text { for } i \in N .
\end{aligned}
$$

Note that $\int_{\Gamma_{i}} \mathbf{n} \cdot \nabla^{\perp} \phi_{j}=\int_{\Gamma_{i}} \boldsymbol{\tau} \cdot \nabla \phi_{j}=0$ for $i \in D$ and $\int_{\Gamma_{i}} \boldsymbol{\tau} \cdot \nabla p_{j}=0$ for $i \in N$ because the integrations are carried out on a closed path.

To see that (2.43) has a solution, note first that it is a singular but consistent system of linear equations. Consider the first two block rows in the upper left of the tableau. Since each $A \nabla p_{i}, A \nabla p_{0 j}$, and $\nabla^{\perp} \phi_{j}$ is divergence free, then $\int_{\Gamma} \mathbf{n} \cdot A \nabla p_{i}=$ $\int_{\Gamma} \mathbf{n} \cdot A \nabla p_{0 i}=\int_{\Gamma} \mathbf{n} \cdot \nabla^{\perp} \phi_{j} 0$. Thus, the sum of any column of these two block rows is zero. The sum of the first two blocks of the right-hand side is also zero by the same reasoning. The null space of the transpose is the same as the null space of the matrix and consists of setting $\alpha_{i}=\alpha$ for $i \in D, \alpha_{0 i}=\alpha$ for $i \in D_{0}$, and $\beta_{i}=0$ for $i \in N$. This corresponds to a constant function, which is in the null space of $\nabla$. A reduced nonsingular system can be found by setting any $\alpha_{i}$ or $\alpha_{0 i}$ to zero and deleting the corresponding row. To see that this reduced system is nonsingular, assume otherwise; then, for some $\mathbf{z}$, there are two solutions whose $\alpha_{i}$ 's differ by something other than a constant; their difference would yield a nonzero function of the form (2.37) that satisfies the hypotheses of Lemma 2.3, which is a contradiction.

With this choice for $\alpha_{0 j}, \alpha_{i}$, and $\beta_{i}$, the function $\mathbf{w}$ satisfies the hypotheses of Lemma 2.3, which implies $\mathbf{w}=\mathbf{0}$. If the form (2.40) had been chosen, a similar argument would yield coefficients $\alpha_{i}, \beta_{0 j}$, and $\beta_{i}$ with one $\beta$ set to zero.

Now suppose Assumption $A 3$ holds. Since the linear system represented by the left-hand side of (2.43) depends only upon $\Omega$ and $A$, then there exist constants $C_{1}-$ $C_{4}$ such that

$$
\begin{aligned}
\max _{j \in D \cup D_{0}}\left|\alpha_{j}\right|+\max _{j \in N \cup N_{0}}\left|\beta_{j}\right| & \leq C_{1}\left(\max _{j \in D \cup D_{0}}\left|\int_{\Gamma_{j}} \mathbf{n} \cdot \mathbf{z}\right|+\max _{j \in N \cup N_{0}}\left|\int_{\Gamma_{i}} \boldsymbol{\tau} \cdot A^{-1} \mathbf{z}\right|\right) \\
& \leq C_{2}\left(\|\mathbf{n} \cdot \mathbf{z}\|_{-1 / 2, \Gamma}+\left\|\boldsymbol{\tau} \cdot A^{-1} \mathbf{z}\right\|_{-1 / 2, \Gamma}\right) \\
& \leq C_{2}\left(\|\mathbf{z}\|_{H(\operatorname{div} ; \Omega)}+\left\|A^{-1} \mathbf{z}\right\|_{H(\text { curl } ; \Omega)}\right) \\
& \leq C_{3}\|\mathbf{z}\|_{0, \Omega} .
\end{aligned}
$$

Finally, (2.30), (2.35), and (2.37) yield

$$
\|\mathbf{z}\|_{1, \Omega} \leq C_{4}\left(\max _{i \in D \cup D_{0}}\left|\alpha_{i}\right|+\max _{i \in N \cup N_{0}}\left|\beta_{i}\right|\right) \leq C\|\mathbf{z}\|_{0, \Omega},
$$

and the lemma is proved.

We remark that the decomposition of $\mathbf{z}$ is not unique. For example, any linear combination of (2.39) and (2.40) whose coefficients sum to one again yields zero.

Proof of Theorem 2.2. We now prove the lower bound in (2.23) by decomposing $\mathbf{v}$ as

$$
\mathbf{v}=A \nabla p+\nabla^{\perp} \phi
$$


where $p, \phi \in H^{2}(\Omega)$. This is done by first choosing $p_{0}$ to satisfy

$$
\left\{\begin{aligned}
\nabla^{*} A \nabla p_{0} & =\nabla^{*} \mathbf{v}, & & \text { in } \quad \Omega, \\
p_{0} & =0, & & \text { on } \quad \Gamma_{D}, \\
\mathbf{n} \cdot A \nabla p_{0} & =0, & & \text { on } \quad \Gamma_{N},
\end{aligned}\right.
$$

which by our assumptions is $H^{2}(\Omega)$ regular and thus (2.20) holds. Together with (2.3) and Assumption $A 3$, this implies that there exist constants $C_{1}$ and $C_{2}$ such that

$$
\left\|A \nabla p_{0}\right\|_{1, \Omega} \leq C_{1}\left\|p_{0}\right\|_{2, \Omega} \leq C_{2}\left\|\nabla^{*} \mathbf{v}\right\|_{0, \Omega} .
$$

Note also that $p_{0}=0$ on $\Gamma_{D}$, which implies $\boldsymbol{\tau} \cdot A^{-1}\left(A \nabla p_{0}\right)=\boldsymbol{\tau} \cdot \nabla p_{0}=0$ on $\Gamma_{D}$. Together with $\mathbf{n} \cdot\left(A \nabla p_{0}\right)=0$ on $\Gamma_{N}$, we see that $A \nabla p_{0} \in \mathbf{W}$.

Next we construct $\phi_{0}$ to satisfy

$$
\left\{\begin{aligned}
\nabla^{*} B \nabla \phi_{0} & =\nabla \times A^{-1} \mathbf{v}, & & \text { in } \Omega, \\
\phi_{0} & =0, & & \text { on } \Gamma_{N}, \\
\mathbf{n} \cdot B \nabla \phi_{0} & =0, & & \text { on } \Gamma_{D} .
\end{aligned}\right.
$$

Again we see that (2.47) is $H^{2}(\Omega)$ regular and there exists a constant $C_{3}$ such that

$$
\left\|\nabla^{\perp} \phi_{0}\right\|_{1, \Omega} \leq\left\|\phi_{0}\right\|_{2, \Omega} \leq C_{3}\left\|\nabla \times A^{-1} \mathbf{v}\right\|_{0, \Omega} .
$$

Moreover, by similar arguments, we have $\nabla^{\perp} \phi_{0} \in \mathbf{W}$.

Now, let

$$
\mathbf{z}=\mathbf{v}-A \nabla p_{0}-\nabla^{\perp} \phi_{0}
$$

Then $\mathbf{z}$ satisfies the hypotheses of Lemma 2.4. Combining (2.49), Lemma 2.4, (2.46), and (2.48) yields

$$
\begin{aligned}
\|\mathbf{v}\|_{1, \Omega} & \leq\|\mathbf{z}\|_{1, \Omega}+\left\|A \nabla p_{0}\right\|_{1, \Omega}+\left\|\nabla^{\perp} \phi_{0}\right\|_{1, \Omega} \\
& \leq C\|\mathbf{z}\|_{0, \Omega}+\left\|A \nabla p_{0}\right\|_{1, \Omega}+\left\|\nabla^{\perp} \phi_{0}\right\|_{1, \Omega} \\
& \leq C\left(\|\mathbf{v}\|_{0, \Omega}+\left\|A \nabla p_{0}\right\|_{0, \Omega}+\left\|\nabla^{\perp} \phi_{0}\right\|_{0, \Omega}\right)+\left\|A \nabla p_{0}\right\|_{1, \Omega}+\left\|\nabla^{\perp} \phi_{0}\right\|_{1, \Omega} \\
& \leq C\|\mathbf{v}\|_{0, \Omega}+C_{4}\left\|A \nabla p_{0}\right\|_{1, \Omega}+C_{5}\left\|\nabla^{\perp} \phi_{0}\right\|_{1, \Omega} \\
& \leq C_{6}\left(\|\mathbf{v}\|_{0, \Omega}+\left\|\nabla^{*} \mathbf{v}\right\|_{0, \Omega}+\left\|\nabla \times A^{-1} \mathbf{v}\right\|_{0, \Omega}\right)
\end{aligned}
$$

for some constant $C_{6}$, and the theorem is proved.

Finally, we remark that the full decomposition of $\mathbf{v}$ takes the form

$$
\mathbf{v}=A \nabla p_{0}+\sum_{j \in D_{0}} \alpha_{0 j} A \nabla p_{0 j}+\sum_{i \in D} \alpha_{i} A \nabla p_{i}+\nabla^{\perp} \phi_{0}+\sum_{j \in N_{0}} \beta_{0 j} \nabla^{\perp} \phi_{0 j}+\sum_{i \in N} \beta_{i} \nabla^{\perp} \phi_{i},
$$

where $\alpha_{i}$ and $\beta_{i}$ are chosen as in Lemma 2.4.

2.2. Three dimensions. Our additional assumptions for $n=3$ restrict the boundary $\Gamma_{0}$ to be either Dirichlet or Neumann; that is, $\Gamma_{0} \subseteq \Gamma_{D}$ or $\Gamma_{0} \subseteq \Gamma_{N}$. Further, $\Gamma_{0}$ is now either $C^{1,1}$ or a convex polyhedron. The results in this section generalize Theorems 3.7, 3.8, and 3.9 in Chapter I of [20], where, in addition to the above restrictions on $\Gamma_{0}$, it is assumed that the entire boundary is either Dirichlet or 
Neumann and that $A=I$. Unlike the two-dimensional proof, we use the result in [20] in our three-dimensional proof, and thus make the same assumptions on $\Gamma_{0}$.

TheOREM 2.3. Let $\Omega \in \Re^{3}$ satisfy Assumption A1. Assume that $A=I$ and that either $\Gamma_{N}=\Gamma$ or $\Gamma_{D}=\Gamma$. Then Theorem 2.2 holds.

Proof. The proof follows from Theorems 3.7 and 3.9 in Chapter I of [20] and Theorem 3.1 in [11].

Given the more general assumptions on $A, \Gamma_{D}$, and $\Gamma_{N}$, the upper bound in (2.23) is immediate, so our task is again to establish the lower bound in (2.23). We first gather some tools. The next two lemmas are technical but essential to what follows.

LEMMA 2.5. Let $\Omega \subset \Re^{3}$ such that the boundary $\partial \Omega$ is piecewise $C^{1,1}$. If $\mathbf{v} \in$ $H(\operatorname{curl} ; \Omega)$ and $\mathbf{n} \times \mathbf{v}=\mathbf{0}$ on $\hat{\Gamma} \subseteq \Gamma$, then $\mathbf{n} \cdot(\nabla \times \mathbf{v})=0$ on $\hat{\Gamma}$ (in the trace sense).

Proof. The proof follows from a modification to Remark 2.5 in [20]. We offer the following heuristic proof. First, since $\mathbf{v} \in H(\mathbf{c u r l} ; \Omega)$, then $\mathbf{n} \times \mathbf{v}$ is well defined on $\Gamma$. Also, $\nabla \times \mathbf{v} \in H(\operatorname{div} ; \Omega)$ so $\mathbf{n} \cdot(\nabla \times \mathbf{v})$ is well defined on $\Gamma$. Now $\mathbf{n} \times \mathbf{v}=\mathbf{0}$ on $\hat{\Gamma}$ implies $\mathbf{v}$ is normal to $\hat{\Gamma}$. Assume that $\mathbf{v} \in \mathcal{D}(\bar{\Omega})$ and let $\mathbf{x} \in \hat{\Gamma}$. Consider the definition

$$
\nabla \times \mathbf{v}(\mathbf{x})=\lim _{\Delta \mathcal{V} \rightarrow 0} \frac{1}{\Delta \mathcal{V}} \int_{\partial \mathcal{V}} \hat{\mathbf{n}} \times \mathbf{v},
$$

where the limit is taken over any convenient neighborhood $\mathcal{V} \subset \Omega$ of $\mathbf{x}$ with volume $\Delta \mathcal{V}$ and surface normal $\hat{\mathbf{n}}$. For example, since $\Gamma$ is piecewise $C^{1,1}$, we may choose cubes with two sides tangent to the boundary, on which $\hat{\mathbf{n}}=\mathbf{n}$, which yields $\mathbf{n} \cdot(\hat{\mathbf{n}} \times \mathbf{v})=$ $\mathbf{v} \cdot(\hat{\mathbf{n}} \times \mathbf{n})=\mathbf{0}$; on the other cube sides, we have the limiting property $\mathbf{n} \cdot(\hat{\mathbf{n}} \times$ $\mathbf{v}) \frac{\Delta \mathcal{A}}{\Delta \mathcal{V}} \rightarrow 0$ since $\mathbf{v}$ is normal to $\partial \Omega$ at $\mathbf{x}$. The result for $\mathbf{v} \in H(\operatorname{curl} ; \Omega)$ follows by continuity.

LEMMA 2.6. Let $\Omega \subset \Re^{3}$ and let $\Gamma$ be a simple, closed, piecewise $C^{1,1}$ surface in $\Omega$. Let $p \in H^{1}(\Omega)$ and $\boldsymbol{\psi} \in H^{1}(\Omega)^{3}$. Then

$$
\int_{\Gamma}((\mathbf{n} \cdot \nabla \times \boldsymbol{\psi}) p+(\mathbf{n} \times \boldsymbol{\psi}) \cdot \nabla p)=0 .
$$

Proof. The proof follows from a modification to Remark 2.5 in [20]. We offer the following heuristic proof. Let $\mathbf{n}=\left(n_{1}, n_{2}, n_{3}\right), \boldsymbol{\psi}=\left(\psi_{1}, \psi_{2}, \psi_{3}\right)$. Then

$\mathbf{n} \times \boldsymbol{\psi}=\left(\begin{array}{ccc}0 & n_{3} & -n_{2} \\ -n_{3} & 0 & n_{1} \\ n_{2} & -n_{1} & 0\end{array}\right)\left(\begin{array}{c}\psi_{1} \\ \psi_{2} \\ \psi_{3}\end{array}\right), \quad \nabla \times \boldsymbol{\psi}=\left(\begin{array}{ccc}0 & \partial_{3} & -\partial_{2} \\ -\partial_{3} & 0 & \partial_{1} \\ \partial_{2} & -\partial_{1} & 0\end{array}\right)\left(\begin{array}{l}\psi_{1} \\ \psi_{2} \\ \psi_{3}\end{array}\right)$.

Notice that each row of the matrix defining $\mathbf{n} \times$ is a vector tangent to the surface, say $\boldsymbol{\tau}_{1}, \boldsymbol{\tau}_{2}, \boldsymbol{\tau}_{3}$. A little algebra on (2.50) yields

$$
\int_{\Gamma}((\mathbf{n} \cdot \nabla \times \boldsymbol{\psi}) p+(\mathbf{n} \times \boldsymbol{\psi}) \cdot \nabla p)=\sum_{i=1}^{3} \int_{\Gamma} \boldsymbol{\tau}_{i} \cdot \nabla\left(\psi_{i} p\right) .
$$

Since the surface is piecewise $C^{1,1}$ and closed, each term in the sum is zero.

The next two theorems summarize results found in [20] that we will need.

THEOREM 2.4. Let $\Omega \subset \Re^{3}$ satisfy Assumption A1.

a. Then

$$
\mathbf{w} \in H(\operatorname{div} ; \Omega), \quad \nabla^{*} \mathbf{w}=0, \quad \int_{\Gamma_{i}} \mathbf{n} \cdot \mathbf{w}=0 \text { for } i=0, \ldots, L
$$


if and only if there exists $\boldsymbol{\psi} \in H(\mathbf{c u r l} ; \Omega)$ such that

$$
\mathbf{w}=\nabla \times \boldsymbol{\psi} .
$$

b. Given (2.51), then there exists $\boldsymbol{\psi} \in H^{1}(\Omega)^{3}$ such that

$$
\mathbf{w}=\nabla \times \boldsymbol{\psi}, \quad \nabla^{*} \boldsymbol{\psi}=0 .
$$

c. Given (2.51), then there exists $\boldsymbol{\psi} \in H(\mathbf{c u r l} ; \Omega)$ such that

$$
\mathbf{w}=\nabla \times \boldsymbol{\psi}, \quad \nabla^{*} \boldsymbol{\psi}=0, \quad \mathbf{n} \cdot \boldsymbol{\psi}=0 \quad \text { on } \Gamma .
$$

If $\Omega$ is simply connected, then $\psi$ is unique. Moreover, if $\Gamma$ is $C^{1,1}$, then $\psi \in H^{1}(\Omega)^{3}$.

d. Given (2.51) and, in addition, $\Omega$ is simply connected and $\mathbf{n} \cdot \mathbf{w}=0$ on $\Gamma$, then there exists a unique $\boldsymbol{\psi} \in H(\mathbf{c u r l} ; \Omega)$ such that

$$
\mathbf{w}=\nabla \times \boldsymbol{\psi}, \quad \nabla^{*} \boldsymbol{\psi}=0, \quad \mathbf{n} \times \boldsymbol{\psi}=\mathbf{0} \text { on } \Gamma, \quad \int_{\Gamma_{i}} \mathbf{n} \cdot \boldsymbol{\psi}=0 \text { for } i=0, \ldots, L .
$$

Moreover, if $\Gamma$ is $C^{1,1}$, then $\boldsymbol{\psi} \in H^{1}(\Omega)^{3}$.

Proof. See Theorems 3.4, 3.5, and 3.6 in Chapter I of [20].

THEOREM 2.5. Let $\Omega \subset \Re^{3}$ satisfy Assumption A1. Assume that either $\mathbf{n} \times \mathbf{w}=\mathbf{0}$ on $\Gamma$ or that $\Omega$ is simply connected. Then $\mathbf{w} \in H(\mathbf{c u r l} ; \Omega)$ and $\nabla \times \mathbf{w}=\mathbf{0}$ if and only if $\mathbf{w}=\nabla p$ for some $p \in H^{1}(\Omega)$ and $p$ is unique up to a constant.

Proof. See Theorem 2.9 in Chapter I of [20].

Next, as in the two-dimensional proof, we provide a result that allows us to declare that a vector in $\mathbf{W}$ is $\mathbf{0}$.

LEMMA 2.7. Let $A$ be uniformly symmetric positive definite on a simply connected $\Omega \subset \Re^{3}$, which satisfies Assumptions A1 and A2. Let $\mathbf{z} \in \mathbf{W}$ satisfy

$$
\begin{aligned}
& \text { i) } \quad \nabla^{*} \mathbf{z}=0, \text { in } \Omega \text {, } \\
& \text { ii) } \nabla \times A^{-1} \mathbf{z}=\mathbf{0}, \text { in } \Omega \text {, } \\
& \text { iii) } \int_{\Gamma_{i}} \mathbf{n} \cdot \mathbf{z}=0, \quad \text { for } i \in D \text {. }
\end{aligned}
$$

Then $\mathbf{z}=\mathbf{0}$.

Proof. If $\Gamma_{0} \subseteq \Gamma_{N}$, then $\mathbf{n} \cdot \mathbf{z}=0$ on $\Gamma_{0}$ and we let $\widehat{N}=N \cup\{0\}, \widehat{D}=D$. If $\Gamma_{0} \subseteq \Gamma_{D}$, let $\widehat{N}=N, \widehat{D}=D \cup\{0\}$. In either case, since $\mathbf{z} \in \mathbf{W}$, then assumptions (2.56) i) and iii) and Theorem 2.4 imply there exists $\boldsymbol{\psi} \in H^{1}(\Omega)^{3}$ such that $\mathbf{z}=\nabla \times \boldsymbol{\psi}$. Assumptions (2.56) ii) and Theorem 2.5 imply there exists $p \in H^{1}(\Omega)$ such that $A^{-1} \mathbf{z}=\nabla p$. Assumption (2.56) i) and Green's formula then yield

$$
\begin{aligned}
0 & =\left(\nabla^{*} \mathbf{z}, p\right) \\
& =(\mathbf{z}, \nabla p)-\int_{\Gamma}(\mathbf{n} \cdot \mathbf{z}) p \\
& =\left(A^{-1} \mathbf{z}, \mathbf{z}\right)-\int_{\Gamma}(\mathbf{n} \cdot \mathbf{z}) p .
\end{aligned}
$$

Since $\mathbf{z} \in \mathbf{W}$ implies $\mathbf{n} \cdot \mathbf{z}=0$ on $\Gamma_{N}$, we then have

$$
\begin{aligned}
\left(A^{-1} \mathbf{z}, \mathbf{z}\right) & =\sum_{i \in \widehat{D}} \int_{\Gamma_{i}}(\mathbf{n} \cdot \mathbf{z}) p+\sum_{i \in \widehat{N}} \int_{\Gamma_{i}}(\mathbf{n} \cdot \mathbf{z}) p \\
& =\sum_{i \in \widehat{D}} \int_{\Gamma_{i}}(\mathbf{n} \cdot \mathbf{z}) p .
\end{aligned}
$$


Likewise, $\mathbf{z} \in \mathbf{W}$ implies $\mathbf{n} \times A^{-1} \mathbf{z}=\mathbf{0}$ on $\Gamma_{D}$, which by Lemma 2.6 yields

$$
\begin{aligned}
\int_{\Gamma_{i}}(\mathbf{n} \cdot \mathbf{z}) p & =\int_{\Gamma_{i}}(\mathbf{n} \cdot \nabla \times \boldsymbol{\psi}) p=-\int_{\Gamma_{i}}(\mathbf{n} \times \boldsymbol{\psi}) \cdot \nabla p \\
& =\int_{\Gamma_{i}}(\mathbf{n} \times \nabla p) \cdot \boldsymbol{\psi}=\int_{\Gamma_{i}}\left(\mathbf{n} \times A^{-1} \mathbf{z}\right) \cdot \boldsymbol{\psi}=0
\end{aligned}
$$

for $i \in \widehat{D}$. Thus,

$$
\left(A^{-1} \mathbf{z}, \mathbf{z}\right)=0 .
$$

Since $A$ is uniformly symmetric positive definite, it follows that $\mathbf{z}=\mathbf{0}$.

The next result is a generalization of Theorem 2.4.

THEOREM 2.6. Let $\Omega \subset \Re^{3}$ be simply connected and satisfy Assumptions $A 1$ and A2. Then

$$
\mathbf{w} \in H(\operatorname{div} ; \Omega), \quad \nabla^{*} \mathbf{w}=0, \quad \mathbf{n} \cdot \mathbf{w}=0 \quad \text { on } \Gamma_{D},
$$

$$
\int_{\Gamma_{i}} \mathbf{n} \cdot \mathbf{w}=0 \text { for } i \in N
$$

if and only if there exists a unique $\boldsymbol{\psi} \in H(\mathbf{c u r l} ; \Omega)$ such that

$$
\begin{gathered}
\mathbf{w}=\nabla \times \boldsymbol{\psi}, \quad \nabla^{*} \boldsymbol{\psi}=0, \quad \mathbf{n} \cdot \boldsymbol{\psi}=0 \text { on } \Gamma_{N}, \\
\mathbf{n} \times \boldsymbol{\psi}=\mathbf{0} \text { on } \Gamma_{D}, \quad \int_{\Gamma_{i}} \mathbf{n} \cdot \boldsymbol{\psi}=0 \text { for } i \in D .
\end{gathered}
$$

If $\Gamma_{0}$ is $C^{1,1}$, then $\boldsymbol{\psi} \in H^{1}(\Omega)^{3}$ and

$$
\|\boldsymbol{\psi}\|_{1, \Omega} \leq C\|\mathbf{w}\|_{0, \Omega},
$$

where $C$ is a constant depending only on $\Omega$.

Proof. First assume that (2.58) holds. Then, by Theorem 2.4a, w $\in H(\operatorname{div} ; \Omega)$, $\nabla^{*} \mathbf{w}=0$, and $\int_{\Gamma_{i}} \mathbf{n} \cdot \mathbf{w}=0$ for $i=1, \ldots, L$. Lemma 2.5 yields $\mathbf{n} \cdot \mathbf{w}=\mathbf{n} \cdot(\nabla \times \boldsymbol{\psi})=0$ on $\Gamma_{D}$.

Now assume that (2.57) holds. We will establish (2.58) and (2.59) together. First suppose that two functions $\boldsymbol{\psi}_{1}$ and $\boldsymbol{\psi}_{2}$ satisfy (2.58). Then it follows from Lemma 2.7 with $A=I$ that $\mathbf{z}=\boldsymbol{\psi}_{1}-\boldsymbol{\psi}_{2}=\mathbf{0}$, which proves the uniqueness. We must now construct $\boldsymbol{\psi} \in H(\operatorname{curl} ; \Omega)$ satisfying $(2.58)$ and, when $\Gamma_{0}$ is $C^{1,1},(2.59)$. To apply Theorem 2.4c, we first extend $\mathbf{w}$ by $\mathbf{0}$ beyond the Dirichlet boundaries according to our two cases:

1) If $\Gamma_{0} \subseteq \Gamma_{N}$, let $\widehat{N}=N \cup\{0\}$ and $\widehat{D}=D$, and define

$$
\widehat{\Omega}=\Omega \cup\left(\cup_{i \in D} \bar{\Omega}_{i}\right) \text {. }
$$

Note that $\partial \widehat{\Omega}=\Gamma_{N}$.

2) If $\Gamma_{0} \subseteq \Gamma_{D}$, let $\mathcal{O}$ be any bounded, open, simply connected set in $\Re^{3}$ with connected $C^{1,1}$ boundary such that $\bar{\Omega} \subset \mathcal{O}$. Let $\Omega_{0}$ be the portion of $\mathcal{O}$ outside $\Gamma_{0}$ (see Fig. 2). Then let $\widehat{N}=N$ and $\widehat{D}=D \cup\{0\}$, and define

$$
\widehat{\Omega}=\Omega_{0} \cup \Gamma_{0} \cup \Omega \cup\left(\cup_{i \in D} \bar{\Omega}_{i}\right) .
$$

Note that $\partial \widehat{\Omega}=\Gamma_{N} \cup \partial \mathcal{O}$. 


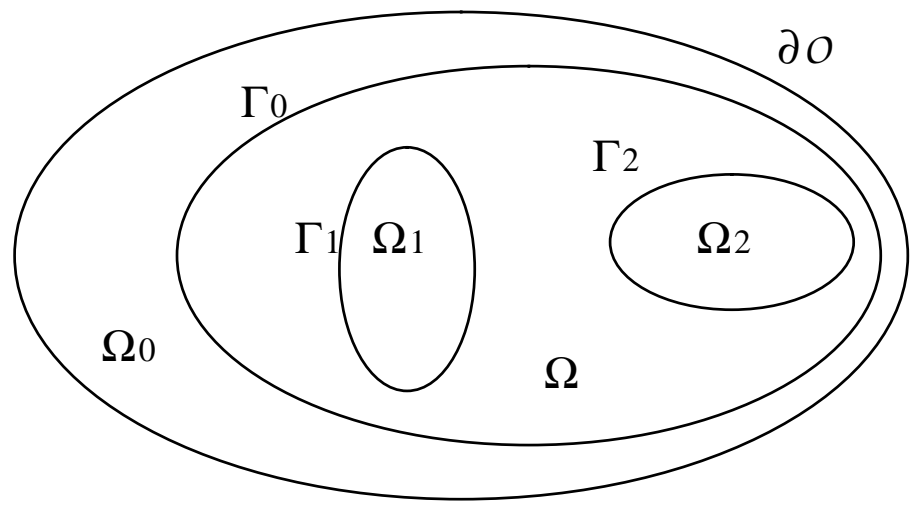

FIG. 2. A section of $\Omega \subset \Re^{3}$.

Now define

$$
\widehat{\mathbf{w}}= \begin{cases}\mathbf{w}, & \text { in } \Omega, \\ \mathbf{0}, & \text { in }(\widehat{\Omega} \backslash \Omega) \cup(\partial \widehat{\Omega} \backslash \partial \Omega) .\end{cases}
$$

Since $\nabla^{*} \widehat{\mathbf{w}} \in L^{2}\left(\Omega_{i}\right)$ and $\mathbf{n} \cdot \widehat{\mathbf{w}}$ is continuous across each $\Gamma_{i}$ for $i \in \widehat{D}$, then $\widehat{\mathbf{w}} \in$ $H(\operatorname{div} ; \widehat{\Omega})$ and $\nabla^{*} \widehat{\mathbf{w}}=0$ in $\widehat{\Omega}$. In either case 1$)$ or 2$), \int_{\Gamma_{i}} \mathbf{n} \cdot \widehat{\mathbf{w}}=0$ on each piece of $\partial \widehat{\Omega}$ by assumption, so Theorem $2.4 \mathrm{c}$ implies that there exists a unique $\widehat{\boldsymbol{\psi}} \in H(\operatorname{curl} ; \widehat{\Omega})$ such that

$$
\widehat{\mathbf{w}}=\nabla \times \widehat{\boldsymbol{\psi}}, \quad \nabla^{*} \widehat{\boldsymbol{\psi}}=0, \quad \mathbf{n} \cdot \widehat{\boldsymbol{\psi}}=0 \quad \text { on } \partial \widehat{\Omega}
$$

and, if $\Gamma_{0}$ is $C^{1,1}$, then $\widehat{\psi} \in H^{1}(\widehat{\Omega})^{3}$.

We now construct $\varphi \in H(\operatorname{curl} ; \widehat{\Omega})$ such that $\nabla^{*} \boldsymbol{\varphi}=0, \nabla \times \boldsymbol{\varphi}=\mathbf{0}$ in $\widehat{\Omega}$, and $\mathbf{n} \times \boldsymbol{\varphi}$ matches $\mathbf{n} \times \widehat{\boldsymbol{\psi}}$ on $\Gamma_{D}$. Notice that $\nabla \times \widehat{\boldsymbol{\psi}}=\mathbf{0}$ in $\Omega_{i}$ for $i \in \widehat{D}$. Since $\Omega$ is simply connected, then each $\Omega_{i}$ is simply connected (cf. proof of Theorem 3.6 in Chapter I of [21]). By Theorem 2.5, $\widehat{\boldsymbol{\psi}}=\nabla q_{i}$ in $\Omega_{i}$, where by (2.60) $q_{i}$ satisfies

$$
\begin{cases}\nabla^{*} \nabla q_{i}=0, & \text { in } \quad \Omega_{i}, \\ \mathbf{n} \cdot \nabla q_{i}=\mathbf{n} \cdot \widehat{\boldsymbol{\psi}}, & \text { on } \quad \Gamma_{i}\end{cases}
$$

for $i \in D$, which is a compatible Neumann problem in $H^{1}\left(\Omega_{i}\right) / \Re$.

If $\Gamma_{0}$ is $C^{1,1}$, then $\widehat{\psi} \in H^{1}(\widehat{\Omega})^{3}$, which implies $\widehat{\boldsymbol{\psi}} \in H^{1}\left(\Omega_{i}\right)^{3}$. Together with the fact that $\Gamma_{i}$ is $C^{1,1}$, we conclude that $\mathbf{n} \cdot \widehat{\boldsymbol{\psi}} \in H^{1 / 2}\left(\Gamma_{i}\right)$. Thus, $(2.61)$ is $H^{2}\left(\Omega_{i}\right)$ regular and

$$
\left\|q_{i}\right\|_{2, \Omega_{i}} \leq M_{i}\|\mathbf{n} \cdot \widehat{\boldsymbol{\psi}}\|_{1 / 2, \Gamma_{i}}
$$

where $M_{i}$ is a constant depending only on $\Omega_{i}$.

For case 2), we need to define the additional function $q_{0}$ that satisfies

$$
\begin{cases}\nabla^{*} \nabla q_{0}=0, & \text { in } \quad \Omega_{0} \\ \mathbf{n} \cdot \nabla q_{0}=\mathbf{n} \cdot \widehat{\boldsymbol{\psi}}, & \text { on } \quad \Gamma_{0} \cup \partial \mathcal{O},\end{cases}
$$


which is a compatible Neumann problem in $H^{1}\left(\Omega_{0}\right) / \Re$. If $\Gamma_{0}$ is $C^{1,1}$, then the same argument as above shows that $(2.63)$ is $H^{2}\left(\Omega_{0}\right)$ regular and

$$
\left\|q_{0}\right\|_{2, \Omega_{0}} \leq M_{0}\|\mathbf{n} \cdot \widehat{\boldsymbol{\psi}}\|_{1 / 2, \Gamma_{0}}
$$

where $M_{0}$ is a constant depending only on $\Omega_{0}$.

Next, we choose $q$ to satisfy

$$
\left\{\begin{aligned}
\nabla^{*} \nabla q & =0, & & \text { in } \quad \\
q & =q_{i}, & & \text { on } \quad \Gamma_{i}, i \in \widehat{D}, \\
\mathbf{n} \cdot \nabla q & =0, & & \text { on } \quad \Gamma_{N} .
\end{aligned}\right.
$$

Then

$$
\widehat{q}= \begin{cases}q, & \text { in } \Omega, \\ q_{i}, & \text { in } \Omega_{i}, i \in \widehat{D},\end{cases}
$$

is in $H^{1}(\widehat{\Omega})$, which implies that $\nabla \widehat{q} \in H(\mathbf{c u r l} ; \widehat{\Omega})$, implying in turn that $\mathbf{n} \times \nabla q_{i}=$ $\mathbf{n} \times \nabla q$ on $\Gamma_{D}$. Thus,

$$
\mathbf{n} \times \widehat{\boldsymbol{\psi}}=\mathbf{n} \times \nabla q_{i}=\mathbf{n} \times \nabla q
$$

on $\Gamma_{D}$.

If $\Gamma_{0}$ is $C^{1,1}$, then each $q_{i} \in H^{2}\left(\Omega_{i}\right)$, which implies that $q_{i} \in H^{3 / 2}\left(\Gamma_{i}\right)$, that problem (2.65) is $H^{2}(\Omega)$ regular, and, using (2.62) and (2.64), that there are constants $C_{1}-C_{3}$ such that

$$
\begin{aligned}
\|q\|_{2, \Omega} & \leq C_{1} \sum_{i \in \widehat{D}}\left\|q_{i}\right\|_{3 / 2, \Gamma_{i}} \leq C_{2} \sum_{i \in \widehat{D}}\left\|q_{i}\right\|_{2, \Omega_{i}} \\
& \leq C_{3} \sum_{i \in \widehat{D}}\|\mathbf{n} \cdot \widehat{\boldsymbol{\psi}}\|_{1 / 2, \Gamma_{i}} \leq C_{3}\|\widehat{\boldsymbol{\psi}}\|_{1, \Omega} .
\end{aligned}
$$

Consider the functions $p_{i}$ for $i \in \widehat{D}$ that satisfy

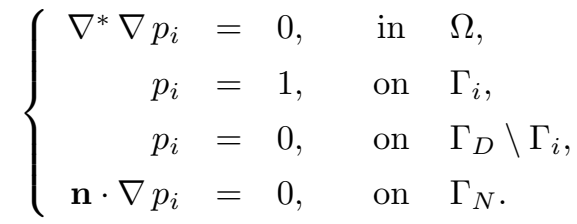

Clearly, (2.68) is $H^{2}(\Omega)$ regular and

$$
\left\|p_{i}\right\|_{2, \Omega} \leq N_{i}
$$

for some constants $N_{i}$ depending only on $\Omega$.

We now set

$$
\boldsymbol{\varphi}=\nabla q+\sum_{i \in \widehat{D}} \alpha_{i} \nabla p_{i}
$$

where the constants $\alpha_{i}$ are chosen so that

$$
\int_{\Gamma_{i}} \mathbf{n} \cdot(\widehat{\psi}-\varphi)=0 \text { for } i \in \widehat{D} .
$$


It is easy to see that $\varphi$ is divergence and curl free in $\Omega$, i.e.,

$$
\nabla^{*} \varphi=0 \text { and } \nabla \times \varphi=0, \quad \text { in } \Omega .
$$

Now the $\alpha_{i}$ are determined by solving the matrix equation

$$
\sum_{j \in \widehat{D}}\left(\int_{\Gamma_{i}} \mathbf{n} \cdot \nabla p_{j}\right) \alpha_{j}=\int_{\Gamma_{i}} \mathbf{n} \cdot(\widehat{\boldsymbol{\psi}}-\nabla q),
$$

for $i \in \widehat{D}$. Thus, $\widehat{\boldsymbol{\psi}}-\nabla q$ and each $\nabla p_{j}$ are divergence free and their normal components vanish on $\Gamma_{N}$, so the integrals of their normal components over the entire boundary are zero. This implies that each column of the system sums to zero, as does the right-hand side. Hence, it is a consistent but singular system, with a null space that contains constant solutions, which yield a zero of $\nabla$. Now if we delete any column and corresponding row, setting the corresponding $\alpha_{i}$ to zero, then Lemma 2.7 with $A=I$ implies that this reduced system is nonsingular: the difference of any two functions arising from (2.70) and (2.73) satisfies the hypotheses of Lemma 2.7, so that the difference is zero; this implies that any solution of the reduced system must be unique; since the system is square, it must be nonsingular.

Since the left-hand side of (2.73) depends only on $\Omega$ (see (2.69)), then there is a constant $C_{4}$ depending only on $\Omega$ such that

$$
\begin{aligned}
\max _{i \in \widehat{D}}\left|\alpha_{i}\right| & \leq C_{4} \sum_{i \in \widehat{D}}\left|\int_{\Gamma_{i}} \mathbf{n} \cdot(\widehat{\boldsymbol{\psi}}-\nabla q)\right| \leq C_{4} \sum_{i \in \widehat{D}}\|\mathbf{n} \cdot(\widehat{\boldsymbol{\psi}}-\nabla q)\|_{-1 / 2, \Gamma_{i}} \\
& \leq C_{4}\|\widehat{\boldsymbol{\psi}}-\nabla q\|_{H(\mathrm{div} ; \Omega)}=C_{4}\|\widehat{\boldsymbol{\psi}}-\nabla q\|_{0, \Omega} .
\end{aligned}
$$

We now define

$$
\psi=\widehat{\psi}-\varphi
$$

To see that $\boldsymbol{\psi}$ satisfies (2.58), note first that (2.60) and (2.72) imply

$$
\nabla^{*} \boldsymbol{\psi}=0 \text { and } \nabla \times \boldsymbol{\psi}=\mathbf{w} \quad \text { in } \Omega .
$$

Equation (2.71) implies that

$$
\int_{\Gamma_{i}} \mathbf{n} \cdot \boldsymbol{\psi}=0
$$

for $i \in \widehat{D}$. Finally, note that (2.60), (2.65), (2.68), and (2.70) yield

$$
\mathbf{n} \cdot \boldsymbol{\psi}=\mathbf{n} \cdot \widehat{\psi}-\mathbf{n} \cdot \varphi=0
$$

on $\Gamma_{N}$, while (2.70), (2.68), and (2.66) yield

$$
\mathbf{n} \times \boldsymbol{\psi}=\mathbf{n} \times \widehat{\boldsymbol{\psi}}-\mathbf{n} \times \nabla q=\mathbf{0}
$$

on $\Gamma_{D}$. 
If $\Gamma_{0}$ is $C^{1,1}$, then, as was mentioned above, $\widehat{\boldsymbol{\psi}} \in H^{1}(\widehat{\Omega})^{3}$. Now, $\widehat{\boldsymbol{\psi}}$ and $\widehat{\Omega}$ were constructed so that $\widehat{\boldsymbol{\psi}} \in H(\operatorname{div}, \widehat{\Omega}) \cap H(\operatorname{curl}, \widehat{\Omega})$ with $\mathbf{n} \cdot \widehat{\boldsymbol{\psi}}=0$ on $\partial \widehat{\Omega}$. Thus, Theorem 3.9 in Chapter I of [20] applies and yields

$$
\begin{aligned}
\|\widehat{\boldsymbol{\psi}}\|_{1, \widehat{\Omega}} & \leq C_{5}\left(\|\widehat{\boldsymbol{\psi}}\|_{0, \widehat{\Omega}}+\left\|\nabla^{*} \widehat{\boldsymbol{\psi}}\right\|_{0, \widehat{\Omega}}+\|\nabla \times \widehat{\boldsymbol{\psi}}\|_{0, \widehat{\Omega}}\right) \\
& =C_{5}\left(\|\widehat{\boldsymbol{\psi}}\|_{0, \widehat{\Omega}}+\|\nabla \times \widehat{\boldsymbol{\psi}}\|_{0, \widehat{\Omega}}\right),
\end{aligned}
$$

for some constant $C_{5}$ depending only on $\widehat{\Omega}$. Lemma 3.6 in Chapter I of [20] yields

$$
\|\widehat{\boldsymbol{\psi}}\|_{0, \widehat{\Omega}} \leq C_{6}\|\nabla \times \widehat{\boldsymbol{\psi}}\|_{0, \widehat{\Omega}}
$$

for some constant $C_{6}$ depending only on $\widehat{\Omega}$. Since $\nabla \times \widehat{\boldsymbol{\psi}}=\mathbf{0}$ in $\widehat{\Omega} \backslash \Omega$, then (2.76) and (2.77) yield

$$
\|\widehat{\boldsymbol{\psi}}\|_{1, \Omega} \leq\|\widehat{\boldsymbol{\psi}}\|_{1, \widehat{\Omega}} \leq C_{7}\|\nabla \times \widehat{\boldsymbol{\psi}}\|_{0, \widehat{\Omega}}=C_{7}\|\mathbf{w}\|_{0, \Omega}
$$

We remark that because of the manner in which $\widehat{\Omega}$ was chosen, the constants $C_{5}-C_{7}$ can be considered to depend only on $\Omega$.

We also have from (2.70), (2.69), (2.74), and (2.67) that

$$
\begin{aligned}
\|\varphi\|_{1, \Omega} & \leq\|\nabla q\|_{1, \Omega}+\sum_{i \in \widehat{D}}\left|\alpha_{i}\right|\left\|\nabla p_{i}\right\|_{1, \Omega} \\
& \leq\|\nabla q\|_{1, \Omega}+C_{8}\|\widehat{\boldsymbol{\psi}}-\nabla q\|_{0, \Omega} \\
& \leq C_{9}\|q\|_{2, \Omega}+C_{8}\|\widehat{\boldsymbol{\psi}}\|_{0, \Omega} \\
& \leq C_{10}\|\widehat{\boldsymbol{\psi}}\|_{1, \Omega},
\end{aligned}
$$

where $C_{8}-C_{10}$ depend only on $\Omega$. Equations (2.78) and (2.79) yield

$$
\|\boldsymbol{\psi}\|_{1, \Omega} \leq\|\widehat{\boldsymbol{\psi}}\|_{1, \Omega}+\|\boldsymbol{\varphi}\|_{1, \Omega} \leq C\|\mathbf{w}\|_{0, \Omega},
$$

which proves the theorem.

Proof of Theorem 2.2. To prove the lower bound in (2.23), we first restrict $\Omega$ to be simply connected and $\Gamma_{0}$ to be $C^{1,1}$, and define $\widehat{D}$ and $\widehat{N}$ as in the proof of Theorem 2.6.

Let $\mathbf{v} \in \mathbf{W}$ and define $\mathbf{w}=\nabla \times A^{-1} \mathbf{v}$. By Theorem $2.4, \mathbf{w} \in H(\operatorname{div} ; \Omega)$ and

$$
\nabla^{*} \mathbf{w}=0, \int_{\Gamma_{i}} \mathbf{n} \cdot \mathbf{w}=0 \text { for } i=0, \ldots, L .
$$

Since $\mathbf{v} \in \mathbf{W}$, then $A^{-1} \mathbf{v} \in H(\operatorname{curl} ; \Omega)$ and $\mathbf{n} \times A^{-1} \mathbf{v}=\mathbf{0}$ on $\Gamma_{D}$, so Lemma 2.5 applied to $A^{-1} \mathbf{v}$ yields

$$
\mathbf{n} \cdot \mathbf{w}=\mathbf{n} \cdot \nabla \times A^{-1} \mathbf{v}=0
$$

on $\Gamma_{D}$. Thus, w satisfies hypothesis (2.57) of Theorem 2.6, so there exists $\boldsymbol{\psi} \in H^{1}(\Omega)^{3}$ such that

$$
\begin{gathered}
\mathbf{w}=\nabla \times \boldsymbol{\psi}, \quad \nabla^{*} \boldsymbol{\psi}=0, \mathbf{n} \cdot \boldsymbol{\psi}=0, \text { on } \Gamma_{N}, \\
\mathbf{n} \times \boldsymbol{\psi}=\mathbf{0} \text { on } \Gamma_{D}, \quad \int_{\Gamma_{i}} \mathbf{n} \cdot \boldsymbol{\psi}=0, \text { for } i \in D
\end{gathered}
$$


Moreover,

$$
\|\boldsymbol{\psi}\|_{1, \Omega} \leq C_{1}\|\mathbf{w}\|_{0, \Omega}=C_{1}\left\|\nabla \times A^{-1} \mathbf{v}\right\|_{0, \Omega} .
$$

Next, consider $A^{-1} \mathbf{v}-\boldsymbol{\psi}$, which is in $H(\operatorname{curl} ; \Omega)$. Since $\nabla \times\left(A^{-1} \mathbf{v}-\boldsymbol{\psi}\right)=\mathbf{0}$, then Theorem 2.5 implies that $A^{-1} \mathbf{v}-\boldsymbol{\psi}=\nabla p$ for some $p \in H^{1}(\Omega)$. We first construct $p$, then bound it. Let $p_{0}$ satisfy

$$
\left\{\begin{aligned}
\nabla^{*} A \nabla p_{0} & =\nabla^{*} \mathbf{v}-\nabla^{*} A \boldsymbol{\psi}, & & \text { in } \quad \Omega, \\
p_{0} & =0, & & \text { on } \Gamma_{D} \\
\mathbf{n} \cdot A \nabla p_{0} & =-\mathbf{n} \cdot A \boldsymbol{\psi}, & & \text { on } \Gamma_{N} .
\end{aligned}\right.
$$

By hypothesis, problem $(2.82)$ is $H^{2}(\Omega)$ regular and, using (2.81), we have

$$
\begin{aligned}
\left\|p_{0}\right\|_{2, \Omega} & \leq C_{2}\left(\left\|\nabla^{*} \mathbf{v}\right\|_{0, \Omega}+\left\|\nabla^{*} A \boldsymbol{\psi}\right\|_{0, \Omega}+\|\mathbf{n} \cdot A \boldsymbol{\psi}\|_{1 / 2, \Gamma_{N}}\right) \\
& \leq C_{2}\left\|\nabla^{*} \mathbf{v}\right\|_{0, \Omega}+C_{3}\|\boldsymbol{\psi}\|_{1, \Omega} \\
& \leq C_{2}\left\|\nabla^{*} \mathbf{v}\right\|_{0, \Omega}+C_{4}\left\|\nabla \times A^{-1} \mathbf{v}\right\|_{0, \Omega} .
\end{aligned}
$$

As in the proof of Theorem 2.6, consider the functions $p_{i}$ for $i \in D$ that satisfy

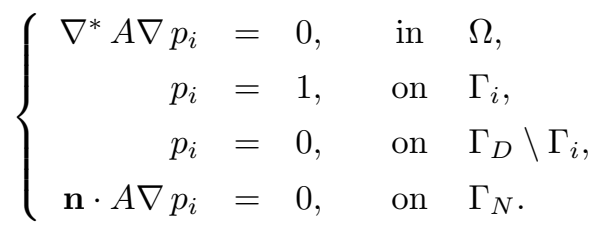

Clearly, (2.84) is $H^{2}(\Omega)$ regular and

$$
\left\|p_{i}\right\|_{2, \Omega} \leq N_{i}
$$

for some constants $N_{i}$ depending only on $\Omega$ and $A$.

Now set

$$
p=p_{0}+\sum_{i \in D} \alpha_{i} p_{i}
$$

and choose the $\alpha_{i}$ so that

$$
\int_{\Gamma_{i}} \mathbf{n} \cdot(\mathbf{v}-A \nabla p-A \psi)=0 \text { for } i \in D .
$$

This yields the matrix equation

$$
\sum_{j \in D}\left(\int_{\Gamma_{i}} A \nabla p_{j}\right) \alpha_{j}=\int_{\Gamma_{i}} \mathbf{n} \cdot\left(\mathbf{v}-A \nabla p_{0}-A \psi\right) \text { for } i \in D .
$$

Similar to the proof of Theorem 2.6, this system has a solution that is either unique (when $D \neq \widehat{D}$ ) or unique up to a constant (when $D=\widehat{D}$ ). The matrix of (2.88) depends only on $\Omega$ and $A$, so there is a constant $C_{5}$ depending only on $\Omega$ and $A$ such 
that

$$
\begin{aligned}
\max _{i \in \widehat{D}}\left|\alpha_{i}\right| & \leq C_{5} \sum_{i \in \widehat{D}}\left|\int_{\Gamma_{i}} \mathbf{n} \cdot\left(\mathbf{v}-A \nabla p_{0}-A \boldsymbol{\psi}\right)\right| \\
& \leq C_{5} \sum_{i \in \widehat{D}}\left\|\mathbf{n} \cdot\left(\mathbf{v}-A \nabla p_{0}-A \boldsymbol{\psi}\right)\right\|_{-1 / 2, \Gamma_{i}} \\
& \leq C_{5}\left\|\mathbf{v}-A \nabla p_{0}-A \boldsymbol{\psi}\right\|_{H(\operatorname{div} ; \Omega)} \\
& \leq C_{5}\|\mathbf{v}\|_{H(\operatorname{div} ; \Omega)}+C_{6}\left\|p_{0}\right\|_{2, \Omega}+C_{7}\|\boldsymbol{\psi}\|_{1, \Omega} .
\end{aligned}
$$

To see that $\mathbf{v}=A \nabla p+A \boldsymbol{\psi}$, set

$$
\mathbf{z}=\mathbf{v}-A \nabla p-A \boldsymbol{\psi},
$$

and note from (2.80) that

$$
\nabla \times A^{-1} \mathbf{z}=\nabla \times A^{-1} \mathbf{v}-\nabla \times \boldsymbol{\psi}=\mathbf{0}
$$

and from (2.82) that

$$
\nabla^{*} \mathbf{z}=\nabla^{*} \mathbf{v}-\nabla^{*} A \nabla p_{0}-\nabla^{*} A \boldsymbol{\psi}=0 .
$$

Since $\mathbf{v} \in \mathbf{W}$ implies $\mathbf{n} \cdot \mathbf{v}=0$ on $\Gamma_{N}$, then (2.82) and (2.84) yield

$$
\begin{aligned}
\mathbf{n} \cdot \mathbf{z} & =\mathbf{n} \cdot \mathbf{v}-\mathbf{n} \cdot A \nabla p-\mathbf{n} \cdot A \boldsymbol{\psi} \\
& =-\mathbf{n} \cdot A \nabla p_{0}-\mathbf{n} \cdot A \boldsymbol{\psi}=0
\end{aligned}
$$

on $\Gamma_{N}$. Likewise, $\mathbf{v} \in \mathbf{W}$ implies $\mathbf{n} \times A^{-1} \mathbf{v}=\mathbf{0}$ on $\Gamma_{D}$ and (2.82) and (2.84) imply $\mathbf{n} \times \nabla p=\mathbf{0}$ on $\Gamma_{D}$. Together with (2.80) we then have

$$
\mathbf{n} \times A^{-1} \mathbf{z}=\mathbf{n} \times A^{-1} \mathbf{v}-\mathbf{n} \times \nabla p-\mathbf{n} \times \boldsymbol{\psi}=\mathbf{0}
$$

on $\Gamma_{D}$. Finally, with $(2.87)$ we see that $\mathbf{z}$ satisfies the hypotheses of Lemma 2.7, so $\mathbf{z}=\mathbf{0}$ and, hence, $\mathbf{v}=A \nabla p+A \psi$.

The bounds (2.85), (2.89), (2.81), and (2.83) yield

$$
\begin{aligned}
\|\mathbf{v}\|_{1, \Omega} & \leq\left\|A \nabla p_{0}\right\|_{1, \Omega}+\sum_{i \in D}\left|\alpha_{i}\right|\left\|A \nabla p_{i}\right\|_{1, \Omega}+\|A \boldsymbol{\psi}\|_{1, \Omega} \\
& \leq\left\|A \nabla p_{0}\right\|_{1, \Omega}+C_{8}\left(\|\mathbf{v}\|_{H(\operatorname{div} ; \Omega)}+\left\|p_{0}\right\|_{2, \Omega}+\|\boldsymbol{\psi}\|_{1, \Omega}\right)+\|A \boldsymbol{\psi}\|_{1, \Omega} \\
& \leq C_{8}\|\mathbf{v}\|_{H(\operatorname{div} ; \Omega)}+C_{9}\left\|p_{0}\right\|_{2, \Omega}+C_{10}\|\boldsymbol{\psi}\|_{1, \Omega} \\
& \leq C\left(\|\mathbf{v}\|_{0, \Omega}+\left\|\nabla^{*} \mathbf{v}\right\|_{0, \Omega}+\left\|\nabla \times A^{-1} \mathbf{v}\right\|_{0, \Omega}\right)
\end{aligned}
$$

for some constants $C_{8}-C_{10}$ and $C$ depending only on $\Omega$ and $A$. This proves the theorem for simply connected $\Omega$ and smooth $\Gamma_{0}$.

The proof for multiply-connected regions follows from a partition of unity argument as in part 2) of the proof of Theorem 3.7 in Chapter I of [21]. The proof when $\Gamma_{0}$ is a convex polyhedron is analogous to the proof for this case offered in Theorem 3.9 in Chapter I of [20]. There, a sequence of subregions $\Omega_{j} \subseteq \Omega$ with $C^{1,1}$ boundaries is constructed to converge outward to $\Omega$. Using the fact that the result holds on each $\Omega_{j}$, the result is shown to hold on $\Omega$.

In the remainder of this paper, we will assume that the conclusion of Theorem 2.2 holds. 
3. Finite element approximation. Using two-dimensional terminology in these last two sections, to discretize the least-squares variational form $(2.14)$, let $T_{h}$ be a regular triangulation of $\Omega$ with elements of size $O(h)$ satisfying the inverse assumption (see [18]). Assume we are given two finite element approximation subspaces

$$
\mathbf{W}_{h} \subset \mathbf{W} \text { and } V_{h} \subset V
$$

defined on the triangulation $T_{h}$. Then the finite element approximation to (2.14) is to find $\left(\mathbf{u}_{h}, p_{h}\right) \in \mathbf{W}_{h} \times V_{h}$ such that

$$
\mathcal{F}\left(\mathbf{u}_{h}, p_{h} ; \mathbf{v}, q\right)=f(\mathbf{v}, q) \quad \forall(\mathbf{v}, q) \in \mathbf{W}_{h} \times V_{h} .
$$

For simplicity, we only consider continuous piecewise linear finite element spaces; i.e.,

$$
V_{h}=\left\{q \in C^{0}(\Omega):\left.q\right|_{K} \in P_{1}(K) \quad \forall K \in T_{h}, q \in V\right\}
$$

and

$$
\mathbf{W}_{h}=\left\{\mathbf{v} \in C^{0}(\Omega)^{n}:\left.v_{l}\right|_{K} \in P_{1}(K) \quad \forall K \in T_{h}, \mathbf{v} \in \mathbf{W}\right\},
$$

where $P_{1}(K)$ is the space of polynomials of degree at most one. Extension of the following results to higher-order finite element approximation spaces is straightforward. (See [11] for the more general case and for the proofs of both theorems of this section.)

THEOREM 3.1. Assume that the solution, $(\mathbf{u}, p)$, of $(2.14)$ is in $H^{1+\alpha}(\Omega)^{n+1}$ for some $\alpha \in[0,1]$, and let $\left(\mathbf{u}_{h}, p_{h}\right) \in \mathbf{W}_{h} \times V_{h}$ be the solution of (3.1). Then

$$
\left\|p-p_{h}\right\|_{1, \Omega}+\left\|\mathbf{u}-\mathbf{u}_{h}\right\|_{1, \Omega} \leq C h^{\alpha}\left(\|p\|_{1+\alpha, \Omega}+\|\mathbf{u}\|_{1+\alpha, \Omega}\right),
$$

where the constant $C$ does not depend on $h, p$, or $\mathbf{u}$.

Proof. The proof is a direct result of Theorem 2.2, Céa's lemma, and interpolation properties of piecewise linear functions (cf. [18]).

Let $\left\{\phi_{1}, \ldots, \phi_{N}\right\}$ and $\left\{\boldsymbol{\psi}_{1}, \ldots, \boldsymbol{\psi}_{M}\right\}$ be bases for $V_{h}$ and $\mathbf{W}_{h}$, respectively. Then, for any $q \in V_{h}$ and $\mathbf{v} \in \mathbf{W}_{h}$, we have

$$
q=\sum_{i=1}^{N} \eta_{i} \phi_{i} \quad \text { and } \quad \mathbf{v}=\sum_{i=1}^{M} \xi_{i} \boldsymbol{\psi}_{i} .
$$

Let $|\boldsymbol{\eta}|$ and $|\boldsymbol{\xi}|$ denote the respective $l_{2}$-norms of the vectors $\boldsymbol{\eta}=\left(\ldots, \eta_{i}, \ldots\right)^{T}$ and $\boldsymbol{\xi}=\left(\ldots, \xi_{i}, \ldots\right)^{T}$. Assume that there exist positive constants $\beta_{i}(i=0,1,2,3)$ such that

$$
\beta_{0} h^{n}|\boldsymbol{\eta}| \leq\|q\|_{0, \Omega} \leq \beta_{1} h^{n}|\boldsymbol{\eta}|
$$

and

$$
\beta_{2} h^{n}|\boldsymbol{\xi}| \leq\|\mathbf{v}\|_{0, \Omega} \leq \beta_{3} h^{n}|\boldsymbol{\xi}| .
$$

Standard finite element spaces with the usual bases satisfy these bounds.

THEOREM 3.2. The condition number of the linear system resulting from (3.1) is $O\left(h^{-2}\right)$.

Proof. The proof is the same as the proof of Theorem 6.1 in [11].

Remark 3.1. The proof of Theorem 6.1 in [11] shows that the smallest and largest eigenvalues of the matrices arising from (3.1) are $O\left(h^{n}\right)$ and $O\left(h^{n-2}\right)$, respectively. It is natural to scale the matrix by multiplying it by a factor of order $h^{-n}$, so that the smallest and largest eigenvalues are $O(1)$ and $O\left(h^{-2}\right)$, respectively. In subsequent sections, we assume that the matrix arising from (3.1) and the operator associated with the bilinear form $\mathcal{F}(\cdot ; \cdot)$ are so scaled. 
4. Multilevel additive and multiplicative algorithms. To develop multilevel solution methods for matrix equations arising from problem (3.1), we start with a coarse grid triangulation $T_{0}$ of $\bar{\Omega}$ having the property that the boundary $\Gamma$ is composed of edges of triangles $K \in T_{0}$ and points of $\bar{\Gamma}_{D} \cap \bar{\Gamma}_{N}$ are included as nodes of $T_{0} . T_{0}$ is then regularly refined several times, giving a family of nested triangulations

$$
T_{0}, T_{1}, \ldots, T_{J}=T_{h}
$$

such that every triangle of $T_{j+1}$ is either a triangle of $T_{j}$ or is generated by subdividing a triangle of $T_{j}$ into some congruent triangles (cf. [18]). Let $h_{j}$ denote the mesh size of triangulation $T_{j}$ and assume that

$$
1<\gamma \leq \gamma_{j} \equiv \frac{h_{j}}{h_{j+1}} \leq C
$$

for fixed constants $\gamma$ and $C$ and for $j=0,1, \ldots, J-1$. (Here and henceforth, we will use $C$ with or without subscripts to denote a generic positive constant independent of the mesh parameter $h$ and the number of levels $J$. We will also use subscripts like $j$ in place of the more cumbersome $h_{j}$ so that $V_{j}$ is used in place of $V_{h_{j}}$, for example.) Without loss of generality, we assume more specifically that

$$
\gamma=\gamma_{j}=2, \quad j=0,1, \ldots, J-1 .
$$

For each $j=0,1, \ldots, J$, we associate the triangulation $T_{j}$ with the continuous piecewise linear finite element space $\mathbf{W}_{j} \times V_{j}$. It is easy to verify that the family of spaces $\left\{\mathbf{W}_{j} \times V_{j}\right\}$ is nested, i.e.,

$$
\mathbf{W}_{0} \times V_{0} \subset \mathbf{W}_{1} \times V_{1} \subset \cdots \subset \mathbf{W}_{J} \times V_{J}=\mathbf{W}_{h} \times V_{h} .
$$

Our multigrid algorithms are described in terms of certain auxiliary operators. Fixing $j \in\{0,1, \ldots, J\}$, define the (self-adjoint) level $j$ operator $\mathcal{F}_{j}: \mathbf{W}_{j} \times V_{j} \longrightarrow$ $\mathbf{W}_{j} \times V_{j}$ for $(\mathbf{u}, p) \in \mathbf{W}_{j} \times V_{j}$ by

$$
\left(\mathcal{F}_{j}(\mathbf{u}, p) ; \mathbf{v}, q\right)=\mathcal{F}(\mathbf{u}, p ; \mathbf{v}, q) \quad \forall(\mathbf{v}, q) \in \mathbf{W}_{j} \times V_{j},
$$

where the inner product $(\cdot ; \cdot)$ is defined by

$$
(\mathbf{u}, p ; \mathbf{v}, q)=(\mathbf{u}, \mathbf{v})_{0, \Omega}+(p, q)_{0, \Omega} .
$$

Also, define the respective elliptic and $L^{2}$ projection operators $\mathcal{P}_{j}, \mathcal{Q}_{j}: \mathbf{W}_{h} \times V_{h} \longrightarrow$ $\mathbf{W}_{j} \times V_{j}$ for $(\mathbf{u}, p) \in \mathbf{W}_{h} \times V_{h}$ by

$$
\mathcal{F}\left(\mathcal{P}_{j}(\mathbf{u}, p) ; \mathbf{v}, q\right)=\mathcal{F}(\mathbf{u}, p ; \mathbf{v}, q) \quad \forall(\mathbf{v}, q) \in \mathbf{W}_{j} \times V_{j}
$$

and

$$
\left(\mathcal{Q}_{j}(\mathbf{u}, p) ; \mathbf{v}, q\right)=(\mathbf{u}, p ; \mathbf{v}, q) \quad \forall(\mathbf{v}, q) \in \mathbf{W}_{j} \times V_{j} .
$$

It is easy to verify that

$$
\mathcal{Q}_{j} \mathcal{F}_{J}=\mathcal{F}_{j} \mathcal{P}_{j}
$$

Let $\mathcal{R}_{0}=\mathcal{F}_{0}^{-1}$ and let $\mathcal{R}_{j}: \mathbf{W}_{j} \times V_{j} \longrightarrow \mathbf{W}_{j} \times V_{j}$ for $1 \leq j \leq J$ denote the smoothing operators. Below we specify certain general conditions on $\mathcal{R}_{j}$. For this purpose, we define $\lambda_{j}$ to be the largest eigenvalue (or spectral radius) of $\mathcal{F}_{j}$ for $1 \leq j \leq J$. 
Problem (3.1) can be rewritten as

$$
\mathcal{F}_{J}\left(\mathbf{u}_{J}, p_{J}\right)=F_{J}
$$

where $F_{J}$ is the right-hand side vector. The additive multigrid preconditioner is defined by

$$
\mathcal{G}_{J}=\sum_{j=0}^{J} \mathcal{R}_{j} \mathcal{Q}_{j} .
$$

The effectiveness of $\mathcal{G}_{J}$ is estimated by the condition number of the preconditioned operator

$$
\mathcal{G}_{J} \mathcal{F}_{J}=\sum_{j=0}^{J} \mathcal{T}_{j},
$$

where

$$
\mathcal{T}_{j}=\mathcal{R}_{j} \mathcal{Q}_{j} \mathcal{F}_{J}
$$

For the multiplicative multigrid scheme, we define the following.

ALGORITHM 4.1 (Multiplicative multigrid algorithm). Given the initial approximation $\left(\mathbf{u}_{J}^{\text {old }}, p_{J}^{\text {old }}\right)$, perform the following steps:

1. Letting $z_{J}=\left(\mathbf{u}_{J}^{\text {old }}, p_{J}^{\text {old }}\right)$, compute an update from each successively coarser level according to

$$
z_{J-j-1}=z_{J-j}+\mathcal{R}_{J-j} \mathcal{Q}_{J-j}\left(F_{J}-\mathcal{F}_{J} z_{J-j}\right) \quad \text { for } \quad j=0,1, \ldots, J-1 .
$$

2. Letting $\hat{z}_{0}=z_{0}$, compute an update from each successively finer level according to

$$
\hat{z}_{j}=\hat{z}_{j-1}+\mathcal{R}_{j-1} \mathcal{Q}_{j-1}\left(F_{J}-\mathcal{F}_{J} \hat{z}_{j-1}\right) \quad \text { for } \quad j=1,2, \ldots, J .
$$

3. The final approximation is

$$
\left(\mathbf{u}_{J}^{\text {new }}, p_{J}^{\text {new }}\right)=\hat{z}_{J} .
$$

Letting $\left(\mathbf{u}_{J}, p_{J}\right)$ denote the exact solution of (4.2), then it is easy to verify that the multiplicative multigrid error equation for the new approximation $\left(\mathbf{u}_{J}^{\text {new }}, p_{J}^{\text {new }}\right)$ is given by

$$
\left(\mathbf{u}_{J}, p_{J}\right)-\left(\mathbf{u}_{J}^{\text {new }}, p_{J}^{\text {new }}\right)=\mathcal{E}_{J}^{s}\left(\left(\mathbf{u}_{J}, p_{J}\right)-\left(\mathbf{u}_{J}^{\text {old }}, p_{J}^{\text {old }}\right)\right),
$$

where the least-squares error reduction operator is given by

$$
\mathcal{E}_{J}^{s}=\mathcal{E}_{J} \mathcal{E}_{J}^{*}
$$

with

$$
\begin{aligned}
& \mathcal{E}_{J}=\left(\mathcal{I}-\mathcal{T}_{J}\right)\left(\mathcal{I}-\mathcal{T}_{J-1}\right) \cdots\left(\mathcal{I}-\mathcal{T}_{0}\right), \\
& \mathcal{E}_{J}^{*}=\left(\mathcal{I}-\mathcal{T}_{0}^{*}\right) \cdots\left(\mathcal{I}-\mathcal{T}_{J-1}^{*}\right)\left(\mathcal{I}-\mathcal{T}_{J}^{*}\right) .
\end{aligned}
$$


(Here we use the fact that the coarsest grid uses an exact solver, so that $\mathcal{I}-\mathcal{T}_{0}=$ $\left(\mathcal{I}-\mathcal{T}_{0}\right)^{2}$.) Note that $\mathcal{E}_{J}^{*}$ is the adjoint of $\mathcal{E}_{J}$ with respect to the inner product $\mathcal{F}(\cdot ; \cdot)$. Since the error reduction operator $\mathcal{E}_{J}^{s}$ is self-adjoint with respect to the inner product $\mathcal{F}(\cdot ; \cdot)$, we have

$$
\left\|\mathcal{E}_{J}^{s}\right\|_{\mathcal{F}}=\left\|\mathcal{E}_{J}\right\|_{\mathcal{F}}^{2}
$$

where the $\mathcal{F}$-norm is defined by

$$
\|\cdot\|_{\mathcal{F}}=\sqrt{\mathcal{F}(\cdot ; \cdot)} .
$$

Remark 4.1. Algorithm 4.1 is a conventional V(1,1)-cycle multigrid algorithm applied to problem (4.2). More general multigrid algorithms, including nonsymmetric cycling schemes like $V(1,0)$-cycles and other cycling schemes like $W$-cycles, may be defined (see [22] and [26]), but we restrict ourselves to the above algorithm for concreteness and because the results are most interesting for this case. The following immediately extends to such schemes, although the $W$-cycle results are not stronger (see [6]). Moreover, our optimal $V$-cycle theory can be used in the usual way (cf. [26]) to establish optimal total complexity of full-multigrid V-cycles (FMV). This is straightforward but nevertheless important: while the $V$-cycle is viewed as an iterative method that obtains optimal algebraic convergence factors, an FMV algorithm based on such cycles is essentially a direct method that achieves overall accuracy to the level of discretization error at a total cost proportional to the number of unknowns.

Letting

$$
\begin{aligned}
\mathcal{G}(\mathbf{u}, p ; \mathbf{v}, q)=(\mathbf{u}, p ; \mathbf{v}, q)+\left(\nabla^{*} \mathbf{u}, \nabla^{*} \mathbf{v}\right)_{0, \Omega} \\
+\left(\operatorname{curl}\left(A^{-1} \mathbf{u}\right), \operatorname{curl}\left(A^{-1} \mathbf{v}\right)\right)_{0, \Omega}+(\nabla p, \nabla q)_{0, \Omega}
\end{aligned}
$$

and

$$
(\mathbf{u}, p ; \mathbf{v}, q)_{1, \Omega}=(\mathbf{u}, p ; \mathbf{v}, q)+(\nabla \mathbf{u}, \nabla \mathbf{v})_{0, \Omega}+(\nabla p, \nabla q)_{0, \Omega},
$$

by Theorems 2.1 and 2.2 we know that the bilinear forms $\mathcal{F}(\cdot ; \cdot)$ and $\mathcal{G}(\cdot ; \cdot)$ and the inner product $(\cdot ; \cdot)_{1, \Omega}$ are uniformly equivalent on $\mathbf{W}_{h} \times V_{h}$ with respect to $h$ and $J$; i.e., there exist positive constants $C_{i}(i=0,1,2,3)$ such that, for any $(\mathbf{v}, q) \in \mathbf{W}_{h} \times V_{h}$,

$$
\begin{aligned}
C_{0}(\mathbf{v}, q ; \mathbf{v}, q)_{1, \Omega} & \leq C_{1} \mathcal{G}(\mathbf{v}, q ; \mathbf{v}, q) \leq \mathcal{F}(\mathbf{v}, q ; \mathbf{v}, q) \\
& \leq C_{2} \mathcal{G}(\mathbf{v}, q ; \mathbf{v}, q) \leq C_{3}(\mathbf{v}, q ; \mathbf{v}, q)_{1, \Omega}
\end{aligned}
$$

Optimality of the additive scheme is a direct consequence of this equivalence and existing multigrid theory (cf. $[28,29,5])$. We state it without proof.

THEOREM 4.1 (Additive multigrid algorithm). For any $(\mathbf{v}, q) \in \mathbf{W}_{h} \times V_{h}$, assume that $\mathcal{R}_{j}$ is a symmetric operator with respect to $(\cdot ; \cdot)$ and that it satisfies

$$
C_{1} \frac{\|(\mathbf{v}, q)\|_{0, \Omega}^{2}}{\lambda_{j}} \leq\left(\mathcal{R}_{j}(\mathbf{v}, q) ; \mathbf{v}, q\right) \leq C_{2} \frac{\|(\mathbf{v}, q)\|_{0, \Omega}^{2}}{\lambda_{j}} \quad \text { for } j=1,2, \ldots, J .
$$

Then

$$
C_{1} \mathcal{F}(\mathbf{v}, q ; \mathbf{v}, q) \leq \mathcal{F}\left(\mathcal{G}_{J} \mathcal{F}_{J}(\mathbf{v}, q) ; \mathbf{v}, q\right) \leq C_{2} \mathcal{F}(\mathbf{v}, q ; \mathbf{v}, q)
$$


Although multiplicative multigrid is often more efficient than the additive scheme, its convergence theory can be more complicated. We will establish convergence of multiplicative multigrid by verifying conditions required by the theory developed in [5] (see also [36]), the only nontrivial task of which is to verify their second assumption on the smoother as the next lemma does.

LEMMA 4.1. Let $\tilde{\mathcal{R}}_{0}=\mathcal{F}_{0}^{-1}, \tilde{\mathcal{R}}_{j}=\frac{1}{\lambda_{j}} \mathcal{I}$ for $j>0$, where $\mathcal{I}$ is the identity operator, and $\tilde{\mathcal{T}}_{j}=\tilde{\mathcal{R}}_{j} \mathcal{Q}_{j} \mathcal{F}_{J}$. Then, for any fixed $\beta \in\left[0, \frac{1}{2}\right)$, we have

$$
\mathcal{F}\left(\tilde{\mathcal{T}}_{j}(\mathbf{v}, q) ; \mathbf{v}, q\right) \leq C\left(\frac{h_{j}}{h_{i}}\right)^{2 \beta} \mathcal{F}(\mathbf{v}, q ; \mathbf{v}, q)
$$

for any $(\mathbf{v}, q) \in \mathbf{W}_{i} \times V_{i}$ with $i \leq j$.

Proof. First note that (see Lemma 4.3 of [5]) for any $\beta \in\left[0, \frac{1}{2}\right), \varphi \in H^{1}(\Omega)^{n+1}$, and $\boldsymbol{\psi} \in H^{1+\beta}(\Omega)^{n+1}$, we have

$$
\left|\int_{\Omega} \frac{\partial \varphi_{s}}{\partial x_{k}} \frac{\partial \psi_{t}}{\partial x_{l}} d x\right| \leq C\left(\eta^{-1}\left\|\varphi_{s}\right\|_{0, \Omega}+\eta^{\frac{\beta}{1-\beta}}\left\|\varphi_{s}\right\|_{1, \Omega}\right)\left\|\psi_{t}\right\|_{1+\beta, \Omega}
$$

for $1 \leq s, t \leq n+1,1 \leq k, l \leq n$, and any $\eta>0$. (It is important to note that continuous piecewise linear functions are in $H^{1+\beta}(\Omega)$ so that $\left\|\psi_{t}\right\|_{1+\beta, \Omega}<\infty$; see [7].) Hence,

$$
|\mathcal{F}(\boldsymbol{\varphi} ; \boldsymbol{\psi})| \leq C\left(\eta^{-1}\|\varphi\|_{0, \Omega}^{2}+\eta^{\frac{\beta}{1-\beta}}\|\varphi\|_{1, \Omega}^{2}\right)^{\frac{1}{2}}\|\boldsymbol{\psi}\|_{1+\beta, \Omega}
$$

Analogous to the proof of Lemma 4.2 in [5], we use the vector inverse inequalities

$$
\begin{aligned}
\|\boldsymbol{\varphi}\|_{1, \Omega} \leq C h_{j}^{-1}\|\boldsymbol{\varphi}\|_{0, \Omega} \quad \forall \boldsymbol{\varphi} \in \mathbf{W}_{j} \times V_{j}, \\
\|\boldsymbol{\psi}\|_{1+\beta, \Omega} \leq C h_{i}^{-\beta}\|\boldsymbol{\psi}\|_{1, \Omega} \quad \forall \boldsymbol{\psi} \in \mathbf{W}_{i} \times V_{i},
\end{aligned}
$$

for any $\beta \in\left[0, \frac{1}{2}\right)$, which follow immediately from the inverse inequalities for scalar functions; see [7]. From the definitions of $\mathcal{F}_{j}$ and $\tilde{\mathcal{T}}_{j}$, we have

$$
\mathcal{F}\left(\tilde{\mathcal{T}}_{j}(\mathbf{v}, q) ; \mathbf{v}, q\right)=\frac{1}{\lambda_{j}} \mathcal{F}\left(\mathcal{F}_{j}(\mathbf{v}, q) ; \mathbf{v}, q\right)=\frac{1}{\lambda_{j}}\left(\mathcal{F}_{j}(\mathbf{v}, q) ; \mathcal{F}_{j}(\mathbf{v}, q)\right) .
$$

For $(\mathbf{v}, q) \in \mathbf{W}_{i} \times V_{i}$ with $i \leq j,(4.11)$ and (4.12) imply

$$
\begin{array}{ll}
\left\|\mathcal{F}_{j}(\mathbf{v}, q)\right\|_{0, \Omega} & =\sup _{(\mathbf{w}, p) \in \mathbf{W}_{j} \times V_{j}} \frac{\left|\left((\mathbf{w}, p) ; \mathcal{F}_{j}(\mathbf{v}, q)\right)\right|}{\|(\mathbf{w}, p)\|_{0, \Omega}}=\sup _{(\mathbf{w}, p) \in \mathbf{W}_{j} \times V_{j}} \frac{|\mathcal{F}((\mathbf{w}, p) ;(\mathbf{v}, q))|}{\|(\mathbf{w}, p)\|_{0, \Omega}} \\
& \leq C\left(\eta^{-1}+C \eta^{\frac{\beta}{1-\beta}} h_{j}^{-2}\right)^{\frac{1}{2}} h_{i}^{-\beta}\|(\mathbf{v}, q)\|_{1, \Omega} .
\end{array}
$$

Setting $\eta=h_{j}^{2-2 \beta}$ and using (4.14) in (4.13) yields (4.10).

Assume that the smoothing operator $\mathcal{R}_{j}$ satisfies the following conditions for $j=0,1, \ldots, J$ and for all $(\mathbf{v}, q) \in \mathbf{W}_{h} \times V_{h}$, where $\mathcal{K}_{j}=I-\mathcal{R}_{j} \mathcal{F}_{j}$ and $\omega \in(0,2)$ :

$$
\begin{aligned}
& \frac{\|(\mathbf{v}, q)\|_{0, \Omega}^{2}}{\lambda_{j}} \leq C\left(\left(I-\mathcal{K}_{j}^{*} \mathcal{K}_{j}\right) \mathcal{F}_{j}^{-1}(\mathbf{v}, q) ; \mathbf{v}, q\right), \\
& \mathcal{F}\left(\mathcal{T}_{j}(\mathbf{v}, q) ; \mathcal{T}_{j}(\mathbf{v}, q)\right) \leq \omega \mathcal{F}\left(\mathcal{T}_{j}(\mathbf{v}, q) ; \mathbf{v}, q\right) .
\end{aligned}
$$


These assumptions, which correspond to $A 4$ and $A 5$ of [5], are easily verified in general for standard smoothers like Jacobi and Gauss-Seidel (cf. [26]).

THEOREM 4.2 (Multiplicative multigrid algorithm). We have

$$
\mathcal{F}\left(\mathcal{E}_{J}(\mathbf{v}, q) ; \mathcal{E}_{J}(\mathbf{v}, q)\right) \leq \gamma \mathcal{F}(\mathbf{v}, q ; \mathbf{v}, q) \quad \forall(\mathbf{v}, q) \in \mathbf{W}_{h} \times V_{h},
$$

where

$$
\gamma=1-\frac{1}{C} \in(0,1)
$$

Proof. Since the boundary of the domain $\Omega$ is $C^{1,1}$ or a convex polyhedron, it is easy to show (cf. $[28,29,5])$ that, for any $(\mathbf{v}, q) \in \mathbf{W}_{h} \times V_{h}$,

$$
(\mathbf{v}, q ; \mathbf{v}, q)_{1, \Omega} \leq C \sum_{j=0}^{J}\left(\tilde{\mathcal{T}}_{j}(\mathbf{v}, q) ; \mathbf{v}, q\right)_{1, \Omega}
$$

Together with Lemma 3.1 in [5] and (4.8), this inequality implies that

$$
\mathcal{F}(\mathbf{v}, q ; \mathbf{v}, q) \leq C \sum_{j=0}^{J} \mathcal{F}\left(\tilde{\mathcal{T}}_{j}(\mathbf{v}, q) ; \mathbf{v}, q\right) .
$$

The theorem now follows from Theorem 3.2 in [5] and Lemma 4.1.

Remark 4.2. Similar results can be obtained for domain decomposition (see [12]) and local mesh refinement methods (see [5]).

\section{REFERENCES}

[1] R. E. BANK, A comparison of two multilevel iterative methods for nonsymmetric and indefinite elliptic finite element equation, SIAM J. Numer. Anal., 18 (1981), pp. 724-743.

[2] P. B. Bochev And M. D. Gunzburger, Accuracy of the least-squares methods for the NavierStokes equations, Comput. Fluids, 22 (1993), pp. 549-563.

[3] J. H. Bramble, Z. Leyk, AND J. E. PASciak, Iterative schemes for non-symmetric and indefinite elliptic boundary value problems, Math. Comp., 60 (1993), pp. 1-22.

[4] J. H. Bramble, R. D. Lazarov, and J. E. Pasciak, A least-squares approach based on a discrete minus one inner product for first order systems, manuscript.

[5] J. H. Bramble And J. E. Pasciak, New estimates for multilevel algorithms including the V-cycle, Math. Comp., 60 (1993), pp. 447-471.

[6] J. H. Bramble, J. E. Pasciak, J. Wang, and J. Xu, Convergence estimates for multigrid algorithms without regularity assumptions, Math. Comp., 57 (1991), pp. 23-45.

[7] J. H. BRAmble, J. E. PASciak, AND J. XU, The analysis of multigrid algorithms with nonnested spaces or non-inherited quadratic forms, Math. Comp., 56 (1991), pp. 1-34.

[8] F. BREzzI, On the existence, uniqueness and approximation of saddle point problems arising from Lagrange multipliers, RAIRO Ser. Anal. Numer., 8 (1974), no. R-2, pp. 129-151.

[9] Z. CAI, Norm estimates of product operators with application to domain decomposition, Appl. Math. Comput., 53 (1993), pp. 251-276.

[10] Z. CAI AND G. LAI, Convergence estimates of multilevel additive and multiplicative algorithms for nonsymmetric and indefinite problems, Numer. Linear Algebra Appl., 3 (1996), pp. 205-220.

[11] Z. Cai, R. D. Lazarov, T. Manteuffel, and S. MCCormick, First-order system least squares for partial differential equations: Part I, SIAM J. Numer. Anal., 31 (1994), pp. 1785-1799.

[12] Z. CAI AND S. MCCormick, Schwarz alternating procedure for elliptic problems discretized by least squares mixed finite elements, manuscript.

[13] G. F. CAREY AND B. N. JiAnG, Least-squares finite element method preconditioner conjugate gradient solution, Internat. J. Numer. Methods Engrg., 24 (1987), pp. 1283-1296. 
[14] C. L. ChANG, Finite element approximation for grad-div type systems in the plane, SIAM J. Numer. Anal., 29 (1992), pp. 452-461.

[15] C. L. Chang, A least-squares finite element method for the Helmholtz equation, Comput. Methods Appl. Mech. Engrg., 83 (1990), pp. 1-7.

[16] T. F. Chen, On the least-squares approximations to compressible flow problems, Numer. Methods Partial Differential Equations, 2 (1986), pp. 207-228.

[17] T. F. Chen And G. J. Fix, Least-squares finite element simulation of transonic flows, Appl. Numer. Math., 2 (1986), pp. 399-408.

[18] P. G. Ciarlet, The Finite Element Method for Elliptic Problems, North-Holland, Amsterdam, 1978.

[19] D. Gilbarg And S. Trudinger, Elliptic Partial Differential Equations of Second Order, Springer-Verlag, New York, 1977.

[20] V. Girault and P. A. Raviart, Finite Element Methods for Navier-Stokes Equations: Theory and Algorithms, Springer-Verlag, New York, 1986.

[21] P. Grisvard, Elliptic Problems in Nonsmooth Domains, Pitman, Boston, MA, 1985.

[22] W. HackBusch, Multi-Grid Methods and Applications, Springer-Verlag, New York, 1985.

[23] B. N. JiAng AND J. Z. CHAI, Least-squares finite element analysis of steady high subsonic plane potential flows, Acta Mech. Sinica, no. 1, 1980, pp. 90-93, (in Chinese); translation: 82N19512\#, Air Force Systems Command, Wright-Patterson AFB, OH, CSS: Foreign Technology Div., FTD-ID(RS) T-0708-81, pp. 53-59.

$[24]$ B. N. Jiang And L. A. Povinelli, Optimal least-squares finite element method for elliptic problems, Comput. Methods Appl. Mech. Engrg., 102 (1993), pp. 194-212.

[25] J. MANDEL, Multigrid convergence for nonsymmetric and indefinite variational problems and one smoothing step, Appl. Math. Comput., 19 (1986), pp. 201-216.

[26] J. Mandel, S. McCormick, And R. Bank, Variational multigrid theory, in Multigrid Methods, S. McCormick, ed., SIAM, Philadelphia, PA, pp. 131-178.

[27] T. A. Manteuffel, S. F. MCCormick, And G. Starke, First-order systems least-squares functionals for elliptic problems with discontinuous coefficients, in Proc. of the Copper Mountain Counference on Multigrid Methods, Copper Mountain, CO, April 3-7, 1995, pp. 535-550.

[28] P. Oswald, On function spaces related to finite element approximation theory, Z. Anal. Anwendungen, 9 (1990), pp. 43-65.

[29] P. Oswald, On discrete norm estimates related to multilevel preconditioners in the finite element method, in Constructive Theory of Functions, in Proc. Internat. Conf. Varna, 1991, K. G. Ivanov, P. Petruskev, B. Sendov, eds., Bulg. Acad. Sci., Sofia, 1992, pp. 203-214.

[30] A. I. Pehlivanov And G. F. Carey, Error estimates for least-square mixed finite elements, RAIRO MMMNA, 28 (1994), pp. 499-516.

[31] A. I. Pehlivanov, G. F. Carey, and R. D. Lazarov, Least squares mixed finite elements for second order elliptic problems, SIAM J. Numer. Anal., 31 (1994), pp. 1368-1377.

[32] H. M. Schey, Div, Grad, Curl, and All That, W. W. Norton and Co., New York, 1973.

[33] G. Strang And G. Fix, An Analysis of the Finite Element Method, Prentice-Hall, Englewood Cliffs, NJ, 1973.

[34] J. WANG, Convergence analysis of multigrid algorithms for non-selfadjoint and indefinite elliptic problems, SIAM J. Numer. Anal., 30 (1993), pp. 275-285.

[35] J. Xu, A new class of iterative methods for nonselfadjoint or indefinite problems, SIAM J. Numer. Anal., 29 (1992), pp. 303-319.

[36] X. ZhANG, Multilevel Schwarz methods, Numer. Math., 63 (1992), pp. 521-539. 